Ursula Hamenstädt

\title{
Bounded cohomology and isometry groups of hyperbolic spaces
}

Received August 5, 2005 and in revised form September 25, 2006

\begin{abstract}
Let $X$ be an arbitrary hyperbolic geodesic metric space and let $\Gamma$ be a countable subgroup of the isometry group Iso $(X)$ of $X$. We show that if $\Gamma$ is non-elementary and weakly acylindrical (this is a weak properness condition) then the second bounded cohomology groups $H_{b}^{2}(\Gamma, \mathbb{R})$, $H_{b}^{2}\left(\Gamma, \ell^{p}(\Gamma)\right)(1<p<\infty)$ are infinite-dimensional. Our result holds for example for any subgroup of the mapping class group of a non-exceptional surface of finite type not containing a normal subgroup which virtually splits as a direct product.
\end{abstract}

\section{Introduction}

A Banach module for a countable group $\Gamma$ is a Banach space $E$ together with a homomorphism of $\Gamma$ into the group of linear isometries of $E$. For every such Banach module $E$ for $\Gamma$ and every $i \geq 1$, the group $\Gamma$ naturally acts on the vector space $L^{\infty}\left(\Gamma^{i}, E\right)$ of bounded functions $\Gamma^{i} \rightarrow E$. If we denote by $L^{\infty}\left(\Gamma^{i}, E\right)^{\Gamma} \subset L^{\infty}\left(\Gamma^{i}, E\right)$ the linear subspace of all $\Gamma$-invariant such functions, then the second bounded cohomology group $H_{b}^{2}(\Gamma, E)$ of $\Gamma$ with coefficients $E$ is defined as the second cohomology group of the complex

$$
0 \rightarrow L^{\infty}(\Gamma, E)^{\Gamma} \stackrel{d}{\rightarrow} L^{\infty}\left(\Gamma^{2}, E\right)^{\Gamma} \stackrel{d}{\rightarrow} \cdots
$$

with the usual homogeneous coboundary operator $d$ (see [M01]). There is a natural homomorphism of $H_{b}^{2}(\Gamma, E)$ into the ordinary second cohomology group $H^{2}(\Gamma, E)$ of $\Gamma$ with coefficients $E$ which in general is neither injective nor surjective.

In this paper we are only interested in the case that $E=\mathbb{R}$ with the trivial $\Gamma$-action or that $E=\ell^{p}(\Gamma)$ for some $p \in(1, \infty)$ with the natural $\Gamma$-action by right translation which assigns to a $p$-summable function $f$ and an element $g \in \Gamma$ the function $g f: h \mapsto f(h g)$.

Since every homomorphism $\rho$ of $\Gamma$ into a countable group $G$ induces a homomorphism $\rho^{*}: H_{b}^{2}(G, \mathbb{R}) \rightarrow H_{b}^{2}(\Gamma, \mathbb{R})$, second bounded cohomology with real coefficients can be used to find obstructions to the existence of interesting homomorphisms $\Gamma \rightarrow G$. The underlying idea is to find conditions on $G$ and $\rho$ which ensure that the image of the map $\rho^{*}$ is "large" (e.g. infinite-dimensional) and conclude that this imposes restrictions on the group $\Gamma$.

U. Hamenstädt: Mathematisches Institut der Universität Bonn, Beringstraße 1, 53115 Bonn, Germany; e-mail: ursula@math.uni-bonn.de 
Two countable groups $\Gamma, G$ are called measure equivalent [G93] if $\Gamma, G$ admit commuting measure preserving actions on a standard infinite measure Borel space with finite measure fundamental domains. Measure equivalence defines an equivalence relation for countable groups [Fu99a]. Monod and Shalom [MS06] showed that for countable groups, vanishing of the second bounded cohomology group with coefficients in the regular representation is preserved under measure equivalence. Thus second bounded cohomology with coefficients in the regular representation provides an obstruction to the existence of a measure equivalence between two given countable groups.

For the application of these ideas it is necessary to obtain information on these bounded cohomology groups. The first and easiest result in this direction is due to B. Johnson (see [I87] for a discussion and references) who showed that the bounded cohomology of amenable groups with real coefficients is trivial. Later Brooks [Br81] found a combinatorial method for the construction of non-trivial real second bounded cohomology classes and used it to show that the second bounded cohomology group of a finitely generated free group is infinite-dimensional.

Fujiwara [F98] investigated the second real bounded cohomology group of a group of isometries of a hyperbolic geodesic metric space. Such a space $X$ admits a geometric boundary $\partial X$. Each isometry of $X$ acts as a homeomorphism on $\partial X$. The limit set of a group $\Gamma$ of isometries of $X$ is the closed $\Gamma$-invariant subset of $\partial X$ of all accumulation points of a fixed $\Gamma$-orbit in $X$. The group $\Gamma$ is called non-elementary if its limit set contains at least three points; then the limit set of $\Gamma$ is in fact uncountable. Using a refinement and an extension of Brooks' method, Fujiwara showed that for a countable non-elementary group $\Gamma$ of isometries of $X$ acting properly discontinuously on $X$ in a metric sense, the kernel of the map $H_{b}^{2}(\Gamma, \mathbb{R}) \rightarrow H^{2}(\Gamma, \mathbb{R})$ is infinite-dimensional [F98]. Bestvina and Fujiwara extended this result further to countable subgroups of $\operatorname{Iso}(X)$ whose actions on $X$ satisfy some weaker properness assumption [BF02]. Their result is for example valid for non-elementary subgroups of the mapping class group of an oriented surface $S$ of finite type and negative Euler characteristic, i.e. for subgroups of the group of isotopy classes of orientation preserving diffeomorphisms of $S$ which are not virtually abelian. As a consequence, the second bounded cohomology group of every non-elementary subgroup of such a mapping class group is infinite-dimensional.

On the other hand, by a result of Burger and Monod [BM99, BM02], for every irreducible lattice $\Gamma$ in a connected semisimple Lie group with finite center, no compact factors and of rank at least 2 the kernel of the natural map $H_{b}^{2}(\Gamma, \mathbb{R}) \rightarrow H^{2}(\Gamma, \mathbb{R})$ vanishes. Together with the results of Fujiwara [F98] and Bestvina and Fujiwara [BF02] it follows easily that the image of every homomorphism of $\Gamma$ into a finitely generated word hyperbolic group or into the mapping class group of an oriented surface of finite type and negative Euler characteristic is finite [BM02, BF02]. The latter result was earlier derived with different methods by Farb and Masur [FM98] building on the work of Kaimanovich and Masur [KM96].

The goal of this note is to present a new method for constructing nontrivial second bounded cohomology classes for a countable group $\Gamma$ from dynamical properties of suitable actions of $\Gamma$. We use it to give a common proof of extensions of the above mentioned results of Fujiwara [F98] and of Bestvina and Fujiwara [BF02], which among other things answers a question raised by Monod and Shalom [MS04, MS06]. 
For the formulation of these results, call a countable group $\Gamma$ of isometries of a (not necessarily proper) hyperbolic geodesic metric space $X$ weakly acylindrical if for every point $x_{0} \in X$ and every $m>0$ there are numbers $R\left(x_{0}, m\right)>0$ and $c\left(x_{0}, m\right)>0$ with the following property. If $x, y \in X$ are such that a geodesic $\gamma$ connecting $x$ to $y$ meets the $m$ neighborhood of $x_{0}$ and if $d(x, y) \geq R\left(x_{0}, m\right)$ then there are at most $c\left(x_{0}, m\right)$ elements $g \in \Gamma$ such that $d(x, g x) \leq m$ and $d(y, g y) \leq m$ (compare with the definition of an acylindrical isometry group in [B03]). We show in Section 4 (see [F98, BF02, MMS04] for earlier results)

Theorem A. Let $\Gamma$ be a non-elementary weakly acylindrical countable group of isometries of an arbitrary hyperbolic geodesic metric space. Then the kernels of the maps $H_{b}^{2}(\Gamma, \mathbb{R}) \rightarrow H^{2}(\Gamma, \mathbb{R})$ and $H_{b}^{2}\left(\Gamma, \ell^{p}(\Gamma)\right) \rightarrow H^{2}\left(\Gamma, \ell^{p}(\Gamma)\right)(1<p<\infty)$ are infinitedimensional.

As an easy corollary of Theorem A and a result of Bowditch [B03] we obtain an extension of the result of Bestvina and Fujiwara [BF02]. For its formulation, we say that a group $\Gamma$ virtually splits as a direct product if $\Gamma$ has a finite index subgroup $\Gamma^{\prime}$ which splits as a direct product of two infinite groups. We show

Corollary B. Let $\Gamma$ be a subgroup of the mapping class group of an oriented surface of finite type and negative Euler characteristic. If $\Gamma$ is not virtually abelian then the kernel of the map $H_{b}^{2}(\Gamma, \mathbb{R}) \rightarrow H^{2}(\Gamma, \mathbb{R})$ is infinite-dimensional. If moreover $\Gamma$ does not contain a normal subgroup which virtually splits as a direct product then the kernel of each of the maps $H_{b}^{2}\left(\Gamma, \ell^{p}(\Gamma)\right) \rightarrow H^{2}\left(\Gamma, \ell^{p}(\Gamma)\right)(1<p<\infty)$ is infinite-dimensional.

The following corollary is an immediate consequence of Corollary B and the work of Burger-Monod and Monod-Shalom [BM99, BM02, MS06]. For its formulation, call a lattice $\Lambda$ in a product $G=G_{1} \times G_{2}$ of two locally compact $\sigma$-compact and non-compact topological groups irreducible if the projection of $\Lambda$ to each of the factors is dense.

Corollary C. Let $\Gamma$ be a subgroup of the mapping class group of an oriented surface of finite type and negative Euler characteristic. Assume that $\Gamma$ does not contain a normal subgroup which virtually splits as a direct product. Then $\Gamma$ is not measure equivalent to an irreducible lattice in a product of two locally compact $\sigma$-compact non-compact topological groups.

For lattices in semisimple Lie groups of higher rank, Corollary C follows from [FM98] and the beautiful work of Furman [Fu99a]. The earlier result of Zimmer [Z91] suffices to deduce Corollary $\mathrm{C}$ for the full mapping class group which admits a linear representation with infinite image. Recently, Kida [K06] derived a much stronger rigidity result. Namely, he showed that for every countable group $\Lambda$ which is measure equivalent to the mapping class group $\mathcal{M}$ of a non-exceptional oriented surface of finite type, there is a homomorphism $\Lambda \rightarrow \mathcal{M}$ with finite kernel and finite index image.

The organization of the paper is as follows. In Section 2, we introduce our method for the construction of second bounded cohomology classes in the concrete example of the fundamental group $\Gamma$ of a convex cocompact complete Riemannian manifold $M$ of 
bounded negative curvature. Such a manifold $M$ contains a compact convex subset $\mathcal{C}(M)$, the so-called convex core, as a strong deformation retract. The group $\Gamma$ is word hyperbolic, and the convex core $\mathcal{C}(M)$ of $M$ is a $K(\Gamma, 1)$-space. Therefore, if $\Gamma$ is non-elementary, i.e. if $\Gamma$ is not abelian, then the dimension of the cohomology group $H^{2}(\Gamma, \mathbb{R})$ is finite, and by Fujiwara's result [F98], the group $H_{b}^{2}(\Gamma, \mathbb{R})$ is infinite-dimensional.

Inspired by a result of Barge and Ghys [BG88], we relate the second bounded cohomology group $H_{b}^{2}(\Gamma, \mathbb{R})$ to the geodesic flow $\Phi^{t}$ of $M$ which acts on the unit tangent bundle $T^{1} M$ of $M$. Since $M$ is convex cocompact, $T^{1} M$ admits a compact $\Phi^{t}$-invariant hyperbolic set $W$ which is the closure of the union of all closed orbits of $\Phi^{t}$. The restriction of $\Phi^{t}$ to $W$ is topologically transitive.

A cocycle for the action of $\Phi^{t}$ on $W$ is a continuous function $c: W \times \mathbb{R} \rightarrow \mathbb{R}$ such that $c(v, s+t)=c(v, t)+c\left(\Phi^{t} v, s\right)$ for all $v \in W$ and all $s, t \in \mathbb{R}$. Two cocycles $c, d$ are cohomologous if there is a continuous function $\psi: W \rightarrow \mathbb{R}$ such that $\psi\left(\Phi^{t} v\right)+c(v, t)=$ $d(v, t)+\psi(v)$. The collection of all cocycles which are cohomologous to a given cocycle $c$ is the cohomology class of $c$. The flip $\mathcal{F}: v \mapsto-v$ acts on $W$ and on the space of cocycles for the geodesic flow preserving cohomology classes. The cohomology class of a cocycle $c$ is called flip anti-invariant if $\mathcal{F}(c)$ is cohomologous to $-c$. We denote by $\mathcal{D C}(M)$ the vector space of all flip anti-invariant cohomology classes of cocycles for the geodesic flow on $W$ which are Hölder continuous, i.e. such that for a fixed number $t>0$ the function $v \mapsto c(v, t)$ is Hölder continuous.

Every smooth closed 1-form on $M$ defines via integration along orbit segments of $\Phi^{t}$ a Hölder continuous cocycle for $\Phi^{t}$ which is anti-invariant under the flip. Two cocycles defined by closed 1-forms in this way are cohomologous if and only if the 1-forms define the same de Rham cohomology class on $M$. Thus $H^{1}(\Gamma, \mathbb{R})=H^{1}(M, \mathbb{R})$ is naturally a subspace of $\mathcal{D C}(M)$. In Section 2 we show

Theorem D. Let $\Gamma$ be the fundamental group of a convex cocompact manifold $M$ of bounded negative curvature. Then the quotient space $\mathcal{D C}(M) / H^{1}(\Gamma, \mathbb{R})$ naturally embeds into $\operatorname{ker}\left(H_{b}^{2}(\Gamma, \mathbb{R}) \rightarrow H^{2}(\Gamma, \mathbb{R})\right)$.

Section 3 contains the main technical result of this paper. Starting from the concrete construction in Section 2, we present an abstract dynamical criterion for infinite-dimensional second bounded cohomology for a countable group $\Gamma$ acting as a group of homeomorphisms on a metric space of bounded diameter. The coefficients of these cohomology groups can be either $\mathbb{R}$ or $\ell^{p}(\Gamma)$ for some $p \in(1, \infty)$. Theorem 4.5 of Section 4 shows that our criterion can be applied to countable groups which admit a non-elementary weakly acylindrical isometric action on a hyperbolic geodesic metric space; this then yields Theorem A. Section 5 contains the proof of Corollary B and Corollary C as well as a short discussion of some applications which are due to Monod and Shalom.

\section{Dynamical cocycles and bounded cohomology}

In this section we consider an $n$-dimensional convex cocompact Riemannian manifold $M$ of bounded negative curvature. Then $M=\tilde{M} / \Gamma$ where $\tilde{M}$ is a simply connected complete Riemannian manifold of bounded negative curvature and $\Gamma$ is a group of isome- 
tries acting properly discontinuously and freely on $\tilde{M}$. The manifold $\tilde{M}$ admits a natural compactification by adding the geometric boundary $\partial \tilde{M}$ which is a topological sphere of dimension $n-1$. Every isometry of $\tilde{M}$ acts on $\partial \tilde{M}$ as a homeomorphism. The limit set $\Lambda$ of $\Gamma$ is the set of accumulation points in $\partial \tilde{M}$ of a fixed orbit $\Gamma x(x \in \tilde{M})$ of the action of $\Gamma$ on $\tilde{M}$. We always assume that the group $\Gamma$ is non-elementary, i.e. that its limit set contains as least three points. Then $\Lambda$ is the smallest nontrivial closed subset of $\partial \tilde{M}$ which is invariant under the action of $\Gamma$.

The geodesic flow $\Phi^{t}$ acts on the unit tangent bundle $T^{1} \tilde{M}$ of $\tilde{M}$ and on the unit tangent bundle $T^{1} M$ of $M$. Let $\tilde{L} \subset T^{1} \tilde{M}$ be the set of all unit tangents of all geodesics with both end-points in $\Lambda$. Then $\tilde{L}$ is invariant under the action of $\Phi^{t}$ and the action of $\Gamma$. The quotient $L=\tilde{L} / \Gamma$ is just the non-wandering set for the action of $\Phi^{t}$ on $T^{1} M$. Since $M$ is convex cocompact by assumption, $L$ is a compact hyperbolic set for the geodesic flow $\Phi^{t}$ on $T^{1} M$. The sets $\tilde{L}$ and $L$ are moreover invariant under the fip $\mathcal{F}: v \mapsto-v$ which maps a unit tangent vector to its negative. The Riemannian metric on $M$ induces a complete Riemannian metric and hence a complete distance function $d$ on $T^{1} M$.

A continuous real-valued cocycle for the action of $\Phi^{t}$ on $L$ is a continuous function $c: L \times \mathbb{R} \rightarrow \mathbb{R}$ with the property that $c(v, t+s)=c(v, t)+c\left(\Phi^{t} v, s\right)$ for all $v \in L$, all $s, t \in \mathbb{R}$. Every continuous function $f: L \rightarrow \mathbb{R}$ defines such a cocycle $c_{f}$ by $c_{f}(v, t)=\int_{0}^{t} f\left(\Phi^{s} v\right) d s$. Two cocycles $b, c$ are called cohomologous if there is a continuous function $\psi: L \rightarrow \mathbb{R}$ such that $\psi\left(\Phi^{t} v\right)+c(v, t)-\psi(v)=b(v, t)$. If $b, c$ are cocycles which are Hölder continuous with respect to the distance $d$ on $L$, i.e. if for fixed $t>0$ the maps $v \mapsto b(v, t)$ and $v \mapsto c(v, t)$ are Hölder continuous, then by Livshits' theorem, $b, c$ are cohomologous if and only if for every periodic point $v$ of the geodesic flow with period $\tau>0$ we have $b(v, \tau)=c(v, \tau)$ [HK95]. Every Hölder continuous cocycle is cohomologous to the cocycle of a Hölder continuous function $f$ (see e.g. [H99]), and two Hölder functions $f, g$ on $L$ are cohomologous, i.e. their cocycles $c_{f}, c_{g}$ are cohomologous, if and only if $\int_{\gamma^{\prime}} f=\int_{\gamma^{\prime}} g$ for every closed geodesic $\gamma$ on $M$ (where $\gamma^{\prime}$ is the unit tangent field of $\gamma$ ).

The flip $\mathcal{F}$ acts on the space of cocycles preserving cohomology classes. We call the cohomology class of a cocycle $c$ anti-invariant under the flip $\mathcal{F}$ if $\mathcal{F}(c)$ is cohomologous to $-c$. If the cohomology class of a Hölder continuous cocycle $c$ is anti-invariant under the flip then there is a Hölder continuous function $f$ which is anti-invariant under the flip, i.e. which satisfies $f(v)=-f(-v)$ for all $v \in L$, such that the cocycle $c_{f}$ defined by $f$ is cohomologous to $c$ (cf. [H97]). Denote by $\mathcal{A}$ the vector space of all Hölder continuous functions $f$ on $L$ which are anti-invariant under the flip $\mathcal{F}$.

Since $L$ is a compact invariant hyperbolic topologically transitive set for the geodesic flow on $T^{1} M$, for every Hölder continuous function $f$ on $L$ and every number $\epsilon_{0}>0$ which is smaller than half of the injectivity radius of $M$ there is a number $k>0$ only depending on the Hölder norm of $f$ with the following property. Let $v, w \in L$ and let $T>0$ be such that $d\left(\Phi^{t} v, \Phi^{t} w\right) \leq \epsilon_{0}$ for all $t \in[0, T]$; then $\left|\int_{0}^{T} f\left(\Phi^{t} v\right) d t-\int_{0}^{T} f\left(\Phi^{t} w\right) d t\right| \leq k$.

A quasi-morphism for $\Gamma$ is a function $\varphi: \Gamma \rightarrow \mathbb{R}$ such that

$$
\|\varphi\|_{0}=\sup _{g, h \in \Gamma}|\varphi(g)+\varphi(h)-\varphi(g h)|<\infty .
$$


The set $\mathcal{Q}$ of all quasi-morphisms for $\Gamma$ naturally has the structure of a vector space. The function \|\|$_{0}: \mathcal{Q} \rightarrow[0, \infty)$ which associates to a quasi-morphism $\varphi$ its defect $\|\varphi\|_{0}$ is a pseudo-norm which vanishes precisely on the subspace of morphisms.

Lemma 2.1. There is a linear map $\Psi: \mathcal{A} \rightarrow \mathcal{Q}$ such that for every $f \in \mathcal{A}$, the defect $\|\Psi(f)\|_{0}$ of $\Psi(f)$ is bounded from above by a constant only depending on the curvature bounds of $M$ and the Hölder norm of $f$.

Proof. Let $f \in \mathcal{A}$ and extend $f$ to a locally Hölder continuous flip anti-invariant function $F$ on $T^{1} M$. Such an extension can be constructed as follows. Choose a probability measure $\mu$ on $L$ for which there are constants $0<a<b$ such that the $\mu$-mass of a ball $B(v, r)$ of radius $r<1$ about a point $v \in L$ is contained in $\left[r^{b}, r^{a}\right]$; for example, the unique measure of maximal entropy for the geodesic flow on $L$ has this property. We view $\mu$ as a probability measure on $T^{1} M$ which is supported in $L$. Let $\tau:[0, \infty) \rightarrow[0,1]$ be a smooth function which satisfies $\tau(t)=1$ for $t$ close to 0 and $\tau[1, \infty)=0$. Via multiplying the restriction of $\mu$ to the metric ball $B(w, r)\left(w \in T^{1} M\right)$ by the function $z \mapsto \tau(d(z, w) / r)$ we may assume that the measures $\mu \mid B(w, r)$ depend continuously on $w \in T^{1} M, r>0$ in the weak*-topology.

For $w \in T^{1} M$ let $\delta(w) \geq 0$ be the distance between $w$ and $L$. For $w \in T^{1} M-L$ define

$$
f_{0}(w)=\frac{1}{\mu(B(w, 2 \delta(w)))} \int_{B(w, 2 \delta(w)) \cap L} f d \mu
$$

and let $f_{0}(w)=f(w)$ for $w \in L$. By assumption on the measures $\mu \mid B(w, r)$ and since $f$ is Hölder continuous, the function $f_{0}$ is locally Hölder continuous and its restriction to $L$ coincides with $f$. Thus we obtain a locally Hölder continuous flip anti-invariant extension $F$ of $f$ to $T^{1} M$ by assigning to $w \in T^{1} M-L$ the value $F(w)=\frac{1}{2}\left(f_{0}(w)-f_{0}(-w)\right)$. For every compact subset $K$ of $T^{1} M$ the Hölder norm of the restriction of $F$ to $K$ only depends on $K$ and on the Hölder norm of $f$. If $F, H$ are the extensions of $f, h$ constructed in this way and if $a, b \in \mathbb{R}$ then $a F+b H$ is the extension of $a f+b h$.

Let again $\Lambda$ be the limit set of $\Gamma$. The closure $\operatorname{Conv}(\Lambda) \subset \tilde{M}$ of the convex hull of $\Lambda$ in $\tilde{M}$ is invariant under the action of $\Gamma$. The convex core $\mathcal{C}(M)=\operatorname{Conv}(\Lambda) / \Gamma$ of $M$ is compact. Let $\tilde{F}$ be the lift of $F$ to $T^{1} \tilde{M}$ and choose a point $p \in \operatorname{Conv}(\Lambda)$. For an element $g \in \Gamma$ define $\Psi(f)(g)$ to be the integral of $\tilde{F}$ over the tangent of the oriented geodesic joining $p$ to $g(p)$. We claim that $\Psi(f)$ is a quasi-morphism for $\Gamma$.

To prove this claim, recall that the curvature of $\tilde{M}$ is pinched between two negative constants and therefore by comparison, for every $\epsilon_{0}>0$ there is a number $k=k\left(\epsilon_{0}\right)>0$ only depending on $\epsilon_{0}$ and an upper curvature bound for $\tilde{M}$ with the following property. Let $T$ be a geodesic triangle in $\tilde{M}$ with vertices $A_{1}, A_{2}, A_{3}$ and denote by $a_{i}$ the side of $T$ connecting $A_{i-1}$ to $A_{i+1}$. Let $q_{i} \in a_{i}$ be the nearest point projection of the vertex $A_{i}$ to the side $a_{i}$ and let $\gamma_{i,+}, \gamma_{i,-}$ be the oriented geodesic arc parametrized by arc length which connects $q_{i}=\gamma_{i,+}(0)$ to $A_{i+1}=\gamma_{i,+}\left(\tau_{i,+}\right)$ and $q_{i}=\gamma_{i,-}(0)$ to $A_{i-1}=\gamma_{i,-}\left(\tau_{i,-}\right)$ (here indices are taken modulo 3 ). Then $t_{i}=\tau_{i+1,+}-\tau_{i,-} \in[-k, k]$ and moreover for every $t \in\left[k, \tau_{i,-}\right]$ the distance between $\gamma_{i,-}^{\prime}(t) \in T^{1} \tilde{M}$ and $\gamma_{i+1,+}^{\prime}\left(t+t_{i}\right) \in T^{1} \tilde{M}$ is at most $\epsilon_{0}$. 
Now by assumption, the function $F$ is anti-invariant under the flip and locally Hölder continuous. Therefore the above discussion implies that the integral of $\tilde{F}$ over the unit tangent field of a closed curve in $\operatorname{Conv}(\Lambda)$ which consists of three geodesic arcs forming a geodesic triangle is bounded from above in absolute value by a universal constant times the Hölder norm of the restriction of $F$ to the compact subset $T^{1} M \mid \mathcal{C}(M)$ of $T^{1} M$ of all unit vectors with foot point in the convex core $\mathcal{C}(M)=\operatorname{Conv}(\Lambda) / \Gamma$. On the other hand, by invariance of $\tilde{F}$ under the action of $\Gamma$ and by anti-invariance of $\tilde{F}$ under the flip, for $g, h \in \Gamma$ the quantity $|\Psi(f)(g)+\Psi(f)(h)-\Psi(f)(g h)|$ is just the absolute value of the integral of $\tilde{F}$ over the unit tangent field of the oriented geodesic triangle in $\operatorname{Conv}(\Lambda) \subset \tilde{M}$ with vertices $p, g(p), g(h(p))$. Thus $\Psi(f)$ is indeed a quasi-morphism and the assignment $f \mapsto \Psi(f)$ defines a linear map $\Psi: \mathcal{A} \rightarrow \mathcal{Q}$. Moreover, the defect $\|\varphi\|_{0}$ of $\varphi$ is bounded from above by a constant only depending on the curvature bounds of $M$ and the Hölder norm of $f$. This shows the lemma.

Two quasi-morphisms $\varphi, \psi$ for $\Gamma$ are called equivalent if $\varphi-\psi$ is a bounded function. This is clearly an equivalence relation. If $\varphi_{1}$ is equivalent to $\varphi_{2}$ and $\psi_{1}$ is equivalent to $\psi_{2}$ then for all $a, b \in \mathbb{R}$ the quasi-morphism $a \varphi_{1}+b \psi_{1}$ is equivalent to $a \varphi_{2}+b \psi_{2}$ and hence the set $\mathcal{Q} B$ of equivalence classes of quasi-morphisms of $\Gamma$ has a natural structure of a vector space. It contains as a subspace the vector space $H^{1}(\Gamma, \mathbb{R})$ of all equivalence classes of morphisms of $\Gamma$. There is an exact sequence

$$
0 \rightarrow H^{1}(\Gamma, \mathbb{R}) \rightarrow \mathcal{Q} B \rightarrow H_{b}^{2}(\Gamma, \mathbb{R}) \rightarrow H^{2}(\Gamma, \mathbb{R})
$$

and therefore the quotient space $\tilde{\mathcal{Q}}=\mathcal{Q} B / H^{1}(\Gamma, \mathbb{R})$ can naturally be identified with the kernel of the map $H_{b}^{2}(\Gamma, \mathbb{R}) \rightarrow H^{2}(\Gamma, \mathbb{R})$ (see [M01]). In particular, an equivalence class of quasi-morphisms can be viewed as a cohomology class of $\Gamma$-invariant bounded cocycles $\varphi \in L^{\infty}\left(\Gamma^{3}, \mathbb{R}\right)^{\Gamma}$. In this interpretation, the cocycle $\varphi$ determined by the quasimorphism $\psi$ associates to a triple $(g, h, u) \in \Gamma^{3}$ the value $\varphi(g, h, u)=\psi\left(g^{-1} h\right)+$ $\psi\left(h^{-1} u\right)-\psi\left(g^{-1} u\right)$.

For $f \in \mathcal{A}$, the definition of the quasi-morphism $\Psi(f)$ in the proof of Lemma 2.1 depends on the choice of an extension of $f$ to a locally Hölder continuous flip antiinvariant function on $T^{1} M$ and also on the choice of a basepoint $p \in \operatorname{Conv}(\Lambda)$. The next lemma shows that the cohomology class of $\Psi(f)$ is independent of these choices.

Lemma 2.2. The cohomology class of the quasi-morphism $\Psi(f)$ does not depend on the choice of the basepoint $p$ nor on the extension of $f$ to a locally Hölder continuous flip anti-invariant function on $T^{1} M$.

Proof. Let $f \in \mathcal{A}$ and let $F$ be a locally Hölder continuous flip anti-invariant extension of $f$ to $T^{1} M$. Denote by $\Psi(f)$ the quasi-morphism constructed in the proof of Lemma 2.1 using the extension $F$ of $f$ and the basepoint $p \in \operatorname{Conv}(\Lambda)$. We first show that a different choice $q \in \operatorname{Conv}(\Lambda)$ of a basepoint gives rise to a quasi-morphism which is equivalent to $\Psi(f)$ in the above sense. For this we follow [BG88]. Let $\tilde{F}$ be the lift of $F$ to $T^{1} \tilde{M}$. For $g \in \Gamma$ define $\rho(g)$ to be the integral of the function $\tilde{F}$ over the unit tangent field of the oriented geodesic quadrangle with vertices $q, g(q), g(p), p$. As in the proof of Lemma 
2.1 we conclude that the function $\rho: \Gamma \rightarrow \mathbb{R}$ is uniformly bounded. Since by invariance of $\tilde{F}$ under the action of $\Gamma$ the integral of $\tilde{F}$ over the oriented geodesic arc connecting $g(q)$ to $g(p)$ is independent of $g \in \Gamma$ and, in particular, it coincides with the negative of the integral of $\tilde{F}$ over the oriented geodesic arc connecting $p$ to $q$, the quasi-morphism defined by $F$ and the basepoint $q$ just equals $\Psi(f)+\rho$. Thus changing the basepoint does not change the equivalence class of the quasi-morphism $\Psi(f)$.

Now we may also replace the point $q \in \operatorname{Conv}(\Lambda)$ by a point $\xi \in \Lambda$. Namely, for $g \in \Gamma$ define $\rho(g)$ to be the oriented integral of the function $\tilde{F}$ over the tangent lines of the ideal geodesic quadrangle with vertices $\xi, g(\xi), g(p), p$. As before, this function is uniformly bounded. By invariance of $\tilde{F}$ under the action of $\Gamma$ and the fact that $\tilde{F}$ is anti-invariant under the flip we find that the 2-cocycle for $\Gamma$ defined as above by the quasi-morphism $\Psi(f)+\rho$ is just the cocycle $\eta \in L^{\infty}\left(\Gamma^{3}, \mathbb{R}\right)^{\Gamma}$ which assigns to a triple $(g, h, u) \in \Gamma^{3}$ the integral of $\tilde{F}$ over the unit tangents of the (possibly degenerate) oriented ideal triangle with vertices $g(\xi), h(\xi), u(\xi)$. Since these unit tangents are contained in the lift $\tilde{L}$ of the non-wandering set $L$ for the geodesic flow on $T^{1} M$, the cocycle $\eta$ only depends on $f$ but not on an extension of $f$ to $T^{1} M$. Thus the cohomology class defined by $\Psi(f)$ is independent of the extension as well.

In the following we denote for $f \in \mathcal{A}$ by $\Theta(f) \in \tilde{\mathcal{Q}}=\operatorname{ker}\left(H_{b}^{2}(\Gamma, \mathbb{R}) \rightarrow H^{2}(\Gamma, \mathbb{R})\right)$ the cohomology class of the quasi-morphism $\Psi(f)$. By Lemma 2.2, this class only depends on $f$. Moreover, the assignment $\Theta: \mathcal{A} \rightarrow \tilde{\mathcal{Q}}$ is clearly linear. We next investigate the kernel of the map $\Theta$.

Since $\Gamma$ is convex cocompact by assumption, there is a natural correspondence between oriented closed geodesics on $M$ and conjugacy classes in $\Gamma$. For every homomorphism $\rho: \Gamma \rightarrow \mathbb{R}$ and every $g \in \Gamma$, the value $\rho(g)$ of $\rho$ on $g$ only depends on the conjugacy class of $g$. Therefore such a homomorphism defines a function on the set of closed geodesics on $M$; we denote the value of $\rho$ on such a closed geodesic $\gamma$ by $\rho(\gamma)$. We have

Lemma 2.3. $\Theta(f)=0$ if and only if there is a morphism $\rho: \Gamma \rightarrow \mathbb{R}$ such that $\int_{\gamma^{\prime}} f=$ $\rho(\gamma)$ for every closed geodesic $\gamma$ on $M$.

Proof. Let $f \in \mathcal{A}$ and assume that there is a morphism $\rho: \Gamma \rightarrow \mathbb{R}$ such that $\int_{\gamma^{\prime}} f=$ $\rho(\gamma)$ for every closed geodesic $\gamma$ on $M$. This morphism defines a class in $H^{1}(M, \mathbb{R})$ and therefore by the de Rham theorem, there is a smooth closed 1-form $\beta$ on $M$ which defines $\rho$ via integration. Let $\tilde{\beta}$ be the pull-back of $\beta$ to a closed 1 -form on $\tilde{M}$. Then $\tilde{\beta}$ is exact and hence the integral of $\tilde{\beta}$ over every piecewise smooth closed curve in $\tilde{M}$ vanishes.

By Livshits' theorem [HK95] and the choice of $\beta$, there is a Hölder continuous flip anti-invariant function $\psi: L \rightarrow \mathbb{R}$ such that $\int_{0}^{T} f\left(\Phi^{t} v\right) d t=\psi\left(\Phi^{T} v\right)+\int_{0}^{T} \beta\left(\Phi^{t} v\right) d t-$ $\psi(v)$ for every $v \in L$ and all $T>0$. As in the proof of Lemma 2.1 we extend $\psi$ to a locally Hölder continuous flip anti-invariant function on all of $T^{1} M$ which we denote by the same symbol. Let $\tilde{\psi}$ be the lift of $\psi$ to $T^{1} \tilde{M}$. Fix a point $p \in \operatorname{Conv}(\Lambda)$ and for $g \in \Gamma$ let $\gamma_{g}$ be the geodesic arc connecting $p=\gamma_{p}(0)$ to $g(p)=\gamma_{p}(T)$. Define $\alpha(g)=\tilde{\psi}\left(\gamma_{g}^{\prime}(T)\right)+\int_{0}^{T} \tilde{\beta}\left(\gamma_{g}^{\prime}(t)\right) d t-\tilde{\psi}\left(\gamma_{g}^{\prime}(0)\right)$. By Lemma 2.1 and Lemma 2.2, $\alpha$ is a 
quasi-morphism for $\Gamma$ which defines the cohomology class $\Theta(f)$. On the other hand, $\alpha$ differs from the quasi-morphism defined by $\beta$ by a bounded function. Since the integral of $\tilde{\beta}$ over every piecewise smooth closed curve in $\tilde{M}$ vanishes, the cohomology class $\Theta(f)$ of the quasi-morphism $\alpha$ vanishes.

On the other hand, let $f \in \mathcal{A}$ and assume that there is no morphism $\rho: \Gamma \rightarrow \mathbb{R}$ such that $\int_{\gamma^{\prime}} f=\rho(\gamma)$ for every closed geodesic $\gamma$ on $M$. We have to show that the cohomology class $\Theta(f)$ does not vanish. By the exact sequence (1) above, this is the case if and only if a quasi-morphism $\Psi(f)$ representing $\Theta(f)$ is not equivalent to any morphism for $\Gamma$.

For this we argue as before. Namely, let $\rho: \Gamma \rightarrow \mathbb{R}$ be any morphism for $\Gamma$ and let $\beta$ be a smooth closed 1-form on $M$ defining $\rho$. By assumption, there is a periodic point $v \in L$ of period $\tau>0$ for the geodesic flow $\Phi^{t}$ such that $\int_{0}^{\tau}(f-\beta)\left(\Phi^{t} v\right) d t=c>0$. Let $\tilde{v}$ be a lift of $v$ to $\tilde{L}$ and let $p \in \tilde{M}$ be the foot-point of $\tilde{v}$. Choose an extension of $f$ to a locally Hölder continuous function $F$ on $T^{1} M$ and let $\tilde{F}$ be the lift of $F$ to $T^{1} \tilde{M}$. By definition, the quasi-morphism $\Psi(f)$ induced by $F$ and the choice of the basepoint $p$ assigns to $g \in \Gamma$ the integral $\int_{0}^{T} \tilde{F}\left(\gamma_{g}^{\prime}(s)\right) d s$ where $\gamma_{g}:[0, T] \rightarrow \tilde{M}$ is the oriented geodesic arc connecting $p$ to $g(p)$. Moreover, this quasi-morphism represents the class $\Theta(f)$. Now let $\eta$ be the geodesic in $\tilde{M}$ which is tangent to $\tilde{v}$. By the choice of $\tilde{v}$ there is an element $h \in \Gamma$ which preserves $\eta$ and whose restriction to $\eta$ is the translation $\eta(t) \mapsto \eta(t+\tau)$ with translation length $\tau$. Hence we have $\Psi(f)\left(h^{m}\right)=m \int_{0}^{\tau} f\left(\Phi^{t} v\right) d t$ and $(\Psi(f)-\rho)\left(h^{m}\right)=m c$ for all $m \in \mathbb{Z}$. In particular, the function $\Psi(f)-\rho$ is unbounded and consequently $\Psi(f)$ is not equivalent to $\rho$. Since $\rho$ was arbitrary this means that the projection of $\Psi(f)$ into $\tilde{\mathcal{Q}}$ does not vanish.

Fix a number $\epsilon_{0}>0$ which is smaller than half of the injectivity radius of $M$ and for $f \in \mathcal{A}$ define $\|f\|_{\mathcal{A}}$ to be the infimum of the numbers $k>0$ with the property that $\left|\int_{0}^{T} f\left(\Phi^{t} v\right) d t-\int_{0}^{T} f\left(\Phi^{t} w\right) d t\right| \leq k$ whenever $v, w \in L$ and $T>0$ are such that $d\left(\Phi^{t} v, \Phi^{t} w\right) \leq \epsilon_{0}$ for every $t \in[0, T]$. We have

Lemma 2.4. \|\|$_{\mathcal{A}}$ is a norm on $\mathcal{A}$.

Proof. We observed above that $\|f\|_{\mathcal{A}}<\infty$ for every Hölder continuous function $f \in$ $\mathcal{A}$. Moreover, we clearly have $\|a f\|_{\mathcal{A}}=|a|\|f\|_{\mathcal{A}}$ for all $f \in \mathcal{A}$ and all $a \in \mathbb{R}$ and $\|f+g\|_{\mathcal{A}} \leq\|f\|_{\mathcal{A}}+\|g\|_{\mathcal{A}}$ by a simple application of the triangle inequality. Thus we are left with showing that $\|f\|_{\mathcal{A}}=0$ only if $f \equiv 0$. For this assume that $0 \not \equiv f \in \mathcal{A}$. Since $f$ is anti-invariant under the flip by assumption, $f$ is not a constant function. Hence by continuity there are points $v, w \in W$ with $d(v, w)<\epsilon_{0} / 2$ and numbers $\delta>0$, $T \in\left(0, \epsilon_{0} / 2\right)$ with $f\left(\Phi^{t} v\right) \geq f\left(\Phi^{t} w\right)+\delta$ for all $t \in[0, T]$. Then $\|f\|_{\mathcal{A}} \geq \delta T$ by the definition of \|\|$_{\mathcal{A}}$.

Call two Hölder functions $f, g \in \mathcal{A}$ weakly cohomologous if $f-g$ is cohomologous to a closed 1-form on $M$, viewed as a function on $T^{1} M$. The class of $f$ under the equivalence relation thus defined will be called the weak cohomology class of $f$. The set $\mathcal{H}$ of weak cohomology classes of Hölder functions is a vector space. For $\psi \in \mathcal{H}$ let $\|\psi\|$ be the 
infimum of the norms $\|f\|_{\mathcal{A}}$ where $f$ runs through all functions in $\mathcal{A}$ which define the weak cohomology class $\psi$. Then \|\| is a pseudo-norm on $\mathcal{H}$.

The Gromov norm $\|\alpha\|$ of an element $\alpha \in H_{b}^{2}(\Gamma, \mathbb{R})$ is the infimum of the supremum norms over all bounded 2-cocycles for $\Gamma$ representing $\alpha$ [G83] (here a bounded 2-cocycle is a bounded $\Gamma$-invariant function on $\Gamma^{3}$ contained in the kernel of the coboundary operator). If $\varphi: \Gamma \rightarrow \mathbb{R}$ is a quasi-morphism then the Gromov norm of the cohomology class defined by $\varphi$ is the infimum of the defects $\|\eta\|_{0}$ where $\eta$ runs through the collection of all quasi-morphisms with $\eta-\varphi$ equivalent to a morphism of $\Gamma$.

By Lemma 2.3, the map $\Theta$ factors through an injective linear map $\mathcal{H} \rightarrow \tilde{\mathcal{Q}}=$ $\operatorname{ker}\left(H_{b}^{2}(\Gamma, \mathbb{R}) \rightarrow H^{2}(\Gamma, \mathbb{R})\right)$ which we denote again by $\Theta$. The following corollary summarizes our discussion and implies Theorem $\mathrm{D}$ from the introduction.

Corollary 2.5. The map $\Theta:(\mathcal{H},\|\|) \rightarrow(\tilde{\mathcal{Q}},\|\|)$ is a continuous embedding.

Proof. By Lemmas 2.1, 2.3 and 2.4 we only have to show the continuity of $\Theta$. For this choose a point $\xi \in \Lambda$. Let $f \in \mathcal{A}$ and let $\tilde{f}$ be the lift of $f$ to $T^{1} \tilde{M}$. For $g, h, u \in \Gamma$ define $\alpha(g, h, u)$ to be the integral of $\tilde{f}$ over the unit tangents of the (possibly degenerate) oriented ideal triangle with vertices $g \xi, h \xi, u \xi$. By Lemma 2.3 and its proof, $\alpha$ is a cocycle which represents the class $\Theta(f)$. The considerations in the proof of Lemma 2.1 show that $|\alpha(g, h, u)| \leq c\|f\|_{\mathcal{A}}$ for a universal constant $c>0$, in particular we have $\alpha \in L^{\infty}\left(\Gamma^{3}, \mathbb{R}\right)^{\Gamma}$ and the Gromov norm of the cohomology class defined by $\alpha$ is not greater than $c\|f\|_{\mathcal{A}}$. From this the continuity of the map $\Theta$ follows.

\section{A dynamical criterion for infinite-dimensional second bounded cohomology}

This section contains the main technical result of this note. We consider an arbitrary countable group $\Gamma$ which acts by homeomorphisms on a metric space $(X, d)$ of finite diameter without isolated points. Our goal is to construct bounded cohomology classes for $\Gamma$ using dynamical properties of the action of $\Gamma$ on $X$ as in Section 2. In the application we have in mind, the space $X$ is the Gromov boundary of a hyperbolic geodesic metric space and $\Gamma$ is a group of isometries acting on $X$ as a group of homeomorphisms.

We begin by describing some properness condition for the action of a countable group $\Gamma$ by homeomorphisms of $(X, d)$. Namely, the metric $d$ on the space $X$ induces a metric on the space $X^{3}$ of triples of points in $X$ which we denote again by $d$; this metric is given by $d\left(\left(x_{1}, x_{2}, x_{3}\right),\left(y_{1}, y_{2}, y_{3}\right)\right)=\sum_{i=1}^{3} d\left(x_{i}, y_{i}\right)$. Let $\Delta \subset X^{3}$ be the closed subset of all triples for which at least two points in the triple coincide. The diagonal action of $\Gamma$ on $X^{3}$ preserves the open set $X^{3}-\Delta$ of triples of pairwise distinct points in $X$.

Definition. The action of $\Gamma$ on $X^{3}-\Delta$ is called metrically proper if for every $v \in(0,1 / 2)$ there are constants $m(v)>0$ and $R(v)>-\log (v / 4)$ such that for any two open disjoint sets $U, V \subset X$ of distance at least $v$ and of diameter at most $e^{-R(v)}$ the following is satisfied.

(1) Let $W \subset X$ be a set of diameter at most $e^{-R(v)}$ whose distance to $U \cup V$ is at least $v$. Write $C=U \times V \times W \subset X^{3}-\Delta$; then for all $k \in \mathbb{Z}$ and every fixed pair of points 
$x_{0} \neq y_{0} \in X$ with $d\left(x_{0}, y_{0}\right) \geq v$ there are at most $m(v)$ elements $g \in \Gamma$ with

$$
\begin{aligned}
g(C) \cap\left\{(x, y, z) \in X^{3}-\Delta \mid\right. & x=x_{0}, y=y_{0}, \\
& \left.e^{-k} \leq \min \left\{d\left(z, x_{0}\right), d\left(z, y_{0}\right)\right\} \leq e^{-k+1}\right\} \neq \emptyset .
\end{aligned}
$$

(2) Let $U^{\prime}, V^{\prime} \subset X$ be open disjoint sets of distance at least $v$ and of diameter at most $e^{-R(v)}$. Let $Z \subset X$ (resp. $\left.Z^{\prime} \subset X\right)$ be the set of all points whose distance to $U \cup V$ (resp. to $U^{\prime} \cup V^{\prime}$ ) is greater than $\nu$. Then there are at most $m(v)$ elements $g \in \Gamma$ with

$$
g(U \times V \times Z) \cap U^{\prime} \times V^{\prime} \times Z^{\prime} \neq \emptyset
$$

If the action of $\Gamma$ on $X^{3}-\Delta$ is metrically proper, then every point in $X^{3}-\Delta$ has a neighborhood $N$ in $X^{3}-\Delta$ such that $g(N) \cap N \neq \emptyset$ only for finitely many $g \in \Gamma$. Namely, for a point $(x, y, z) \in X^{3}-\Delta$ choose $v>0$ sufficiently small that $\min \{d(x, y), d(x, z), d(z, y)\}$ $\geq 2 v$. For this $v$ let $R(v)>0$ be as in the definition of a metrically proper action and let $N$ be the open $e^{-R(v)}$-neighborhood of $(x, y, z)$ in $X^{3}$; then $N \cap g N \neq \emptyset$ only for finitely many $g \in \Gamma$ by the second part of the above definition. Since $X$ does not have isolated points this implies that the quotient $\left(X^{3}-\Delta\right) / \Gamma$ is a metrizable Hausdorff space.

For every $g \in \Gamma$, the fixed point set $\operatorname{Fix}(g)$ for the action of $g$ on $X$ is a closed subset of $X$. The boundary $A(g)$ of the open subset $X^{3}-\operatorname{Fix}(g)^{3}$ of $X^{3}$ is a closed (possibly empty) nowhere dense subset of $X^{3}$. By the above observation, every point $(x, y, z) \in X^{3}-\Delta$ admits a neighborhood $N$ which intersects only finitely many of the sets $A(g)(g \in \Gamma)$. Since $X$ does not have isolated points, the set $X^{3}-\Delta-\bigcup_{g \in \Gamma} A(g)$ is open and dense in $X^{3}-\Delta$. The restriction of the natural projection

$$
\pi: T=X^{3}-\Delta \rightarrow Y=\left(X^{3}-\Delta\right) / \Gamma
$$

to the open dense set $X^{3}-\Delta-\bigcup_{g \in \Gamma} A(g)$ is a local homeomorphism.

The involution $\iota: X^{3} \rightarrow X^{3}$ defined by $\iota(a, b, c)=(b, a, c)$ is an isometry with respect to the metric $d$ on $X^{3}$ induced from the metric on $X$, and its fixed point set is contained in the closed set $\Delta \subset X^{3}$. Thus the restriction of $\iota$ to $T$ acts freely, and it commutes with the diagonal action of $\Gamma$. In particular, $\iota$ naturally acts on $Y$ as a continuous involution and the quotient $Z=Y / \iota$ is a metrizable Hausdorff space. There is an open dense subset of $T$ such that the restriction of the natural projection $\pi_{0}: T \rightarrow Z$ to this set is a local homeomorphism.

For $x \in X$ and $\epsilon>0$ denote by $B(x, \epsilon) \subset X$ the open ball of radius $\epsilon$ about $x$. We next recall the well known notion of north-south dynamics for a homeomorphism of $X$.

Definition. A homeomorphism $g$ of $X$ has north-south dynamics with respect to an attracting fixed point $a \in X$ and a repelling fixed point $b \in X-\{a\}$ if the following is satisfied.

(1) For every $\epsilon>0$ there is a number $m>0$ such that $g^{m}(X-B(b, \epsilon)) \subset B(a, \epsilon)$ and $g^{-m}(X-B(a, \epsilon)) \subset B(b, \epsilon)$.

(2) There is a number $\delta>0$ such that $\bigcup_{m \in \mathbb{Z}} g^{m}(X-B(a, \delta)-B(b, \delta))=X-\{a, b\}$. 
We call a the attracting and $b$ the repelling fixed point of $g$, and $(a, b)$ is the ordered pair of fixed points for $\mathrm{g}$.

The next definition formalizes the idea that the dynamics of each element of a group $G$ of homeomorphisms of $(X, d)$ is uniformly similar to north-south dynamics on a metrically large scale.

Definition. The action of an arbitrary group $G$ on a metric space $(X, d)$ of finite diameter is called weakly hyperbolic if for every $\epsilon>0$ there is $a b=b(\epsilon) \in(0,1)$ with the following property. Let $x, y \in X$ with $d(x, y) \geq 2 \epsilon$ and let $g \in G$ be such that $d(g x, g y) \geq 2 \epsilon$. Let $z \in X-\{x, y\}$ be such that $\min \{d(g z, g x), d(g z, g y)\} \geq \epsilon$. Then $d(g w, g x) \leq d(z, y)^{b} / b$ for every $w \in X$ with $d(w, x) \leq \epsilon$.

Let again $\Gamma$ be a countable group which admits an action on a metric space $(X, d)$ of finite diameter without isolated points by homeomorphisms such that the diagonal action on $T=X^{3}-\Delta$ is metrically proper. Using the above notations, let $C \subset T$ be an open set whose closure $\bar{C}$ has positive distance to $\Delta$ and is mapped by the projection $\pi_{0}: T \rightarrow Z=Y / \iota$ homeomorphically into $Z$. This means that for every $g \in \Gamma$, either $g$ fixes $\bar{C} \cup \iota \bar{C}$ pointwise or $g(\bar{C} \cup \iota \bar{C}) \cap(\bar{C} \cup \iota \bar{C})=\emptyset$. We assume that $C$ is of the form $C=U \times V \times W$ where $U, V, W \subset X$ are open and pairwise of positive distance, say the distance between any two of these sets is at least $4 v>0$. For $R(v)>0$ as in the definition of a metrically proper action we also assume that the diameter of $C$ is smaller than $e^{-R(v)}$. Let $\mathcal{H}_{C}$ be the vector space of all Hölder continuous functions $f: T \rightarrow \mathbb{R}$ supported in $C$. This means that for every $f \in \mathcal{H}_{C}$ there is some $\alpha \in(0,1)$ and some $q>0$ such that $|f(x)-f(y)| \leq q d(x, y)^{\alpha}$ for all $x, y \in C$.

The following lemma is the analogue of Lemma 2.1. For its formulation, denote by $\mathcal{Q}$ the vector space of all quasi-morphisms of $\Gamma$.

Lemma 3.1. Let $(X, d)$ be a metric space of finite diameter without isolated points. Let $\Gamma$ be a countable group which admits a weakly hyperbolic action by homeomorphisms of $X$ such that the action of $\Gamma$ on $T=X^{3}-\Delta$ is metrically proper. Then for every open set $C \subset T$ whose closure projects homeomorphically into $Z=(T / \Gamma) / \iota$ there is a linear map $\Psi: \mathcal{H}_{C} \rightarrow \mathcal{Q}$.

Proof. Using the above notations, write $C=U \times V \times W$ where $U, V, W \subset X$ are open and pairwise of distance at least $4 v>0$. Assume that the diameter of $C$ is at most $e^{-R(v)}$. The product structure of $T$ defines a natural foliation $\mathcal{F}$ on $T$ by requiring that the leaf of $\mathcal{F}$ through $(a, b, c) \in T$ equals the set $F(a, b)=\{(a, b, d) \mid d \in X-\{a, b\}\}$. Thus a leaf of $\mathcal{F}$ is determined by two distinct points in $X$, and the leaf $F(a, b)$ determined by $a \neq b \in X$ can naturally be identified with $X-\{a, b\}$. The foliation $\mathcal{F}$ is invariant under the action of $\Gamma$ and hence it projects to a foliation $\mathcal{F}_{0}$ on $Y=T / \Gamma$.

Let $\mu_{W}$ be a Borel probability measure on $W$ which is positive on open sets. Choose a non-trivial Hölder continuous function $\psi: X \times X \rightarrow[0,1]$ supported in $U \times V$ and let $\mu_{\mathcal{F}}$ be the family of $\Gamma$-invariant $\iota$-invariant Borel measures on the leaves of $\mathcal{F}$ which is determined by the requirement that for every $(u, v) \in U \times V$ the restriction of $\mu_{\mathcal{F}}$ to $F(u, v) \cap C \sim(u, v) \times W$ equals $\psi(u, v) \mu_{W}$. 
We divide the proof of the lemma into two steps. As a notational convention, for $x \in X$ and $\epsilon>0$ denote as before by $B(x, \epsilon)$ the open $\epsilon$-ball about $x$.

Step 1. In the first step we construct, for a given Hölder continuous function $f \in \mathcal{H}_{C}$ supported in $C$, a function $\Psi(f): \Gamma \rightarrow \mathbb{R}$. For this recall that by the choice of $C$, every $g \in \Gamma$ either fixes $\bar{C} \cup \iota \bar{C}$ pointwise or we have $g(\bar{C} \cup \iota \bar{C}) \cap(\bar{C} \cup \iota \bar{C})=\emptyset$. Therefore every function $f \in \mathcal{H}_{C}$ uniquely determines a continuous $\Gamma$-invariant $\iota$-anti-invariant function $\tilde{f}$ on $T$ which is supported in $\bigcup_{g \in \Gamma} g(C \cup \iota C)$ and whose restriction to $C$ coincides with $f$. This means that $\tilde{f}(\iota x)=-\tilde{f}(x)$ for all $x \in T, \tilde{f}(g x)=\tilde{f}(x)$ for all $g \in \Gamma$ and the restriction of $\tilde{f}$ to $C$ coincides with $f$. We claim that for all $x \neq y \in X$ and any neighborhoods $A$ of $x$ and $B$ of $y$ we have $\int_{F(x, y)-A-B}|\tilde{f}| d \mu_{\mathcal{F}}<\infty$, where as before we identify the leaf $F(x, y)$ of the foliation $\mathcal{F}$ with the set $X-\{x, y\}$.

For this consider first the case that $d(x, y) \geq 2 v$ where $v>0$ is as above determined by the choice of the set $C$. Let $k_{0} \geq 1$ be the smallest integer which is not smaller than $-\log v$. If $z \in X$ is such that $d(x, z) \leq e^{-k_{0}}$ then $d(x, z)=\min \{d(x, z), d(y, z)\}$ and hence by the first requirement in the definition of a metrically proper action, for every $k \geq k_{0}$ the number of elements $g \in \Gamma$ with $g(C \cup \iota C) \cap\left(F(x, y) \cap\left(B\left(x, e^{-k}\right)-\right.\right.$ $\left.\left.B\left(x, e^{-k-1}\right)\right)\right) \neq \varnothing$ is bounded from above by a constant $m(v)>0$ only depending on $v$ but not on $k$ and $(x, y)$. Since $\tilde{f}$ is invariant under the action of $\Gamma$ and supported in $\bigcup_{g \in \Gamma} g(C \cup \iota C)$ and since the measures $\mu_{\mathcal{F}}$ are invariant under the action of $\Gamma$ this implies that

$$
\int_{F(x, y) \cap\left(B\left(x, e^{-k}\right)-B\left(x, e^{-k-1}\right)\right)}|\tilde{f}| d \mu_{\mathcal{F}} \leq m(v) \sup \{|f(z)| \mid z \in C\}
$$

for every $k \geq k_{0}$. The same estimate also holds for the analogous integral over $F(x, y) \cap$ $\left(B\left(y, e^{-k}\right)-B\left(y, e^{-k-1}\right)\right)$ provided that $k \geq k_{0}$.

Similarly, since the diameter of $X$ is finite, the set $F(x, y)-B\left(x, e^{-k_{0}}\right)-B\left(y, e^{-k_{0}}\right)$ is the union of finitely many subsets of the form

$$
\left\{z \mid e^{-k_{0}+m-1} \leq \min \{d(z, x), d(z, y)\} \leq e^{-k_{0}+m}\right\} \quad(m \geq 1) .
$$

Using once more the definition of a metrically proper action we conclude that the number of elements $g \in \Gamma$ such that $g(C \cup \iota C) \cap\left(F(x, y)-B\left(x, e^{-k_{0}}\right)-B\left(y, e^{-k_{0}}\right)\right) \neq \varnothing$ is bounded from above by a constant only depending on $\nu$. In particular, the integral $\int_{F(x, y)-B\left(x, e^{-k_{0}}\right)-B\left(y, e^{-k_{0}}\right)}|\tilde{f}| d \mu_{\mathcal{F}}$ is bounded above by a universal multiple of the supremum norm of $f$. Together we conclude that for any neighborhoods $A$ of $x$ and $B$ of $y$ the integral $\int_{F(x, y)-A-B}|\tilde{f}| d \mu_{\mathcal{F}}$ exists, i.e. the claim holds true whenever $d(x, y) \geq 2 v$.

Now let $x \neq y \in X$ be arbitrary points such that the support of $\tilde{f}$ intersects the leaf $F(x, y)$. Since $\tilde{f}$ is supported in $\bigcup_{g \in \Gamma} g(C \cup \iota C)$, there is then an element $g \in \Gamma$ with $d(g x, g y) \geq 2 \nu$. By invariance of $\tilde{f}$ and $\mu_{\mathcal{F}}$ under the action of $\Gamma$, for any neighborhoods $A$ of $x$ and $B$ of $y$ we have

$$
\int_{F(x, y)-A-B}|\tilde{f}| d \mu_{\mathcal{F}}=\int_{F(g x, g y)-g A-g B}|\tilde{f}| d \mu_{\mathcal{F}}
$$


where $g A$ and $g B$ are neighborhoods of $g x$ and $g y$ respectively. Thus indeed $\int_{F(x, y)-A-B}|f| d \mu_{\mathcal{F}}<\infty$ for any two points $x \neq y \in X$ and any neighborhoods $A$ of $x$ and $B$ of $y$, which shows the above claim.

Recall that $C=U \times V \times W$ for open disjoint subsets $U, V, W$ of $X$. Fix a point $x \in U$ and let $A \subset U$ be a small closed metric ball centered at $x$. For $f \in \mathcal{H}_{C}$ and $g \in \Gamma$ such that $g x \neq x$ define

$$
\Psi(f)(g)=\int_{F(x, g x)-A-g(A)} \tilde{f} d \mu_{\mathcal{F}}
$$

and if $g x=x$ then define $\Psi(f)(g)=0$. By the above considerations, the integral (2) exists and hence it defines a function $\Psi(f): \Gamma \rightarrow \mathbb{R}$. Moreover, the assignment $\Psi$ : $f \in \mathcal{H}_{C} \mapsto \Psi(f)$ is a linear map from the vector space $\mathcal{H}_{C}$ into the vector space of all functions on $\Gamma$.

Step 2. In a second step, we show that for every $f \in \mathcal{H}_{C}$ the function $\Psi(f): \Gamma \rightarrow \mathbb{R}$ is a quasi-morphism, i.e. $\sup _{g, h}\{|\Psi(f)(g)+\Psi(f)(h)-\Psi(f)(g h)|\}<\infty$. Observe that by invariance under $\Gamma$, for $g, h \in \Gamma$ we have

$$
\begin{aligned}
\Psi(f)(g)+\Psi(f)(h)-\Psi(f)(g h)= & \int_{F(x, g x)-A-g A} \tilde{f} d \mu_{\mathcal{F}}+\int_{F(g x, g h x)-g A-g h A} \tilde{f} d \mu_{\mathcal{F}} \\
& -\int_{F(x, g h x)-g h A-A} \tilde{f} d \mu_{\mathcal{F} .}
\end{aligned}
$$

Since $f$ is anti-invariant under the involution $\iota$ it is therefore enough to show that there is a number $c(v, f)$ only depending on $v$ and the Hölder norm of $f$ with the following property. Let $\left(x_{1}, x_{2}, x_{3}\right) \in T$ and let $A_{i}$ be any neighborhood of $x_{i}$ in $X(i=1,2,3)$; then

$$
\left|\int_{F\left(x_{1}, x_{2}\right)-A_{1}-A_{2}} \tilde{f} d \mu_{\mathcal{F}}+\int_{F\left(x_{2}, x_{3}\right)-A_{2}-A_{3}} \tilde{f} d \mu_{\mathcal{F}}+\int_{F\left(x_{3}, x_{1}\right)-A_{3}-A_{1}} \tilde{f} d \mu_{\mathcal{F}}\right|<c(\nu, f) .
$$

For this recall that for $g, h \in \Gamma$ the sets $g \bar{C}, h \bar{C}, g(\overline{\iota C}), h(\overline{\iota C})$ either coincide or are disjoint. Moreover, if $\tilde{f} \mid F(y, z) \not \equiv 0$ for some $y \neq z \in X$ then there is some $g \in \Gamma$ such that $d(g y, g z) \geq 2 v$. Define $\mathcal{G}=\left\{g \in \Gamma \mid \max _{i, j \leq 3} d\left(g x_{i}, g x_{j}\right) \geq 2 v\right\}$ and

$$
\mathcal{G}_{0}=\left\{g \in \mathcal{G} \mid \min \left\{d\left(g x_{i}, g x_{j}\right) \mid i \neq j \in\{1,2,3\}\right\} \geq v\right\}
$$

and for $i=1,2,3$ define

$$
\mathcal{G}_{i}=\left\{g \in \mathcal{G} \mid d\left(g x_{i}, g x_{i+1}\right)<v\right\}
$$

(indices are taken modulo 3). By the triangle inequality and the definition of the set $\mathcal{G}$, the sets $\mathcal{G}_{i}(i=0, \ldots, 3)$ are pairwise disjoint and their union equals $\mathcal{G}$.

If $g \in \Gamma$ is such that $(C \cup \iota C) \cap g F\left(x_{i}, x_{i+1}\right) \neq \emptyset$ then $d\left(g x_{i}, g x_{i+1}\right) \geq 2 v$ and therefore $g \in \mathcal{G}_{0}$ if $\min \left\{d\left(g x_{i-1}, g x_{i}\right), d\left(g x_{i-1}, g x_{i+1}\right)\right\} \geq v, g \in \mathcal{G}_{i-1}$ if $d\left(g x_{i-1}, g x_{i}\right)<v$ and $g \in \mathcal{G}_{i+1}$ otherwise (where indices are again taken modulo 3 ). Thus by invariance of $\tilde{f}$ and $\mu_{\mathcal{F}}$ under the action of $\Gamma$ and by the fact that an element $g \in \Gamma$ either fixes $C$ point- 
wise or is such that $g C \cap C=\emptyset$ it is enough to show that there is a number $c_{1}(\nu, f)>0$ only depending on $v$ and the Hölder norm of $f$ such that for $i=0, \ldots, 3$ we have

$$
\sum_{g \in \mathcal{G}_{i}}\left|\sum_{j=1}^{3} \int_{g\left(F\left(x_{j}, x_{j+1}\right)-A_{j}-A_{j+1}\right) \cap(C \cup \mathcal{C})} \tilde{f} d \mu_{\mathcal{F}}\right| \leq c_{1}(\nu, f) .
$$

We first establish the estimate (3) for $i=0$. The case $\mathcal{G}_{0}=\emptyset$ is trivial, so assume that there is some $h \in \mathcal{G}_{0}$ with the additional property that $h\left(F\left(x_{j}, x_{j+1}\right)-A_{j}-A_{j+1}\right) \cap C \neq \varnothing$ for some $j \in\{1,2,3\}$. Recall that $C=U \times V \times W$ where the diameter of the sets $U, V, W$ is at most $e^{-R(v)}<v / 4$. Let $Z \subset X$ be the set of all points whose distance to $U \cup V$ is at least $v$. Then $h\left(x_{j}, x_{j+1}, x_{j+2}\right) \in U \times V \times Z$ and therefore if $u \in \mathcal{G}_{0}$ is another element with $u\left(F\left(x_{j}, x_{j+1}\right)-A_{j}-A_{j+1}\right) \cap C \neq \emptyset$ then $u h^{-1}(U \times V \times Z) \cap U \times V \times Z \neq \emptyset$. Using the second property in the definition of a metrically proper action we conclude that the number of elements $u \in \mathcal{G}_{0}$ with this property is bounded from above by a constant only depending on $v$. The same argument also applies to elements $g \in \mathcal{G}_{0}$ with $g\left(F\left(x_{j}, x_{j+1}\right)-A_{j}-A_{j+1}\right) \cap \iota C \neq \emptyset$ for some $j \in\{1,2,3\}$. As a consequence, for $i=0$ the number of non-zero terms in the sum $(3)$ is bounded from above by a universal constant and the estimate (3) holds true for $i=0$. Thus by symmetry in $i \in\{1,2,3\}$ and by invariance under the action of $\Gamma$ it now suffices to show the estimate (3) for $i=3$.

By definition, for $g \in \mathcal{G}_{3}$ we have $d\left(g x_{1}, g x_{3}\right)<v$ and therefore $g F\left(x_{1}, x_{3}\right) \cap$ $(C \cup \iota C)=\emptyset$. This means that

$$
\begin{aligned}
& \sum_{g \in \mathcal{G}_{3}}\left|\sum_{j=1}^{3} \int_{g\left(F\left(x_{j}, x_{j+1}\right)-A_{j}-A_{j+1}\right) \cap(C \cup \iota C)} \tilde{f} d \mu_{\mathcal{F}}\right| \\
& =\sum_{g \in \mathcal{G}_{3}}\left|\int_{g\left(F\left(x_{1}, x_{2}\right)-A_{2}-A_{3}\right) \cap(C \cup \iota C)} \tilde{f} d \mu_{\mathcal{F}}+\int_{g\left(F\left(x_{2}, x_{3}\right)-A_{3}-A_{1}\right) \cap(C \cup \iota C)} \tilde{f} d \mu_{\mathcal{F}}\right| .
\end{aligned}
$$

By assumption, the action of $\Gamma$ on $X$ is weakly hyperbolic. Thus there is a constant $b>0$ depending on $v$ such that for all $(x, y) \in X \times X$ with $d(x, y) \geq 2 v$, for all $k \geq-\log v$ and for all $z \in X$ with $d(z, x) \leq v$ we have $d(g z, g x) \leq e^{-k b} / b$ whenever $g \in \Gamma$ is such that $(C \cup \iota C) \cap g\left(F(x, y) \cap B\left(y, e^{-k}\right)\right) \neq \emptyset$. In particular, for every $w \in W$ the distance between $(g x, g y, w)$ and $(g z, g y, w)=\iota(g y, g z, w)$ is at most $e^{-k b} / b$.

Now $\tilde{f}$ is a $\Gamma$-invariant $\iota$-anti-invariant function on $T$ which is supported in $\cup_{g \in \Gamma} g(C \cup \iota C)$ and whose restriction $f$ to $C$ satisfies $|f(v)-f(w)| \leq q d(v, w)^{\alpha}$ for some $\alpha, q>0$ and for all $v, w \in C$. Moreover, $\mu_{\mathcal{F}}$ is a $\iota$-invariant $\Gamma$-invariant family of measures on the leaves of $\mathcal{F}$ whose restriction to $C$ is of the form $\psi \mu_{W}$ for a Hölder continuous function $\psi$ supported in $U \times V$. As a consequence of the above discussion on the effect of weak hyperbolicity we conclude that there is a number $\beta>0$ depending only on $v$ and the Hölder norm of $f$ with the following property. Let $x, y \in X$ with $d(x, y) \geq 2 v$; if for some $k \geq-\log v$ the element $g \in \Gamma$ is such that $(C \cup \iota C) \cap g\left(F(x, y) \cap\left(B\left(y, e^{-k}\right)-B\left(y, e^{-k-1}\right)\right)\right) \neq \emptyset$ then for every $z \in X$ with $d(z, x) \leq v$ and every neighborhood $A$ of $y$ we have

$$
\left|\int_{g(F(x, y)-A) \cap(C \cup \iota C)} \tilde{f} d \mu_{\mathcal{F}}+\int_{g(F(y, z)-A) \cap(C \cup \ell C)} \tilde{f} d \mu_{\mathcal{F}}\right| \leq e^{-k \beta} / \beta .
$$


By the first property in the definition of a metrically proper action there is a constant $c>0$ only depending on $v$ such that for all $x, y \in X$ with $d(x, y) \geq 2 v$ and every $k \geq-\log v$ there are at most $c$ elements $g \in \Gamma$ with $(C \cup \iota C) \cap g\left(F(x, y) \cap\left(B\left(y, e^{-k}\right)-\right.\right.$ $\left.\left.B\left(y, e^{-k-1}\right)\right)\right) \neq \emptyset$. Together with the estimate $[5$ we conclude that there is a constant $c_{2}(v, f)>0$ which only depends on $v$ and on the Hölder norm of $f$ with the following property. For $x, y \in X$ with $d(x, y) \geq 2 v$, every $z \in X-\{x, y\}$ with $d(x, z) \leq v$ and every neighborhood $A$ of $y$ we have

$$
\sum_{\{g \in \Gamma \mid d(g x, g z)<\nu\}}\left|\int_{g((F(x, y)-A) \cup(F(y, z)-A)) \cap(C \cup \iota C)} \tilde{f} d \mu \mathcal{F}\right|<c_{2}(\nu, f) .
$$

Now if $g \in \mathcal{G}_{3}$ is such that $g F\left(x_{1}, x_{2}\right) \cap(C \cup \iota C) \neq \emptyset$ then with $y_{i}=g x_{i}$ we have $d\left(y_{1}, y_{3}\right)<v, d\left(y_{1}, y_{2}\right) \geq 2 v$. For any other $h \in \mathcal{G}_{3}$ with $h F\left(x_{1}, x_{2}\right) \cap(C \cup \iota C) \neq \emptyset$ we obtain $d\left(h g^{-1} y_{1}, h g^{-1} y_{2}\right) \geq 2 v$ and $d\left(h g^{-1} y_{1}, h g^{-1} y_{3}\right) \leq v$. By invariance of $\tilde{f}$ and $\mu_{\mathcal{F}}$ under the action of $\Gamma$, inequality (3) above now follows from the estimate (6) and the equation (4).

As a consequence, for every $f \in \mathcal{H}_{C}$ the function $\Psi(f)$ on $\Gamma$ is indeed a quasimorphism. By construction, the assignment $f \mapsto \Psi(f)$ is moreover linear. This completes the proof of the lemma.

In Lemma 3.1 we constructed a linear map $\Psi$ from the vector space $\mathcal{H}_{C}$ onto a vector space $\Psi\left(\mathcal{H}_{C}\right) \subset \mathcal{Q}$ of quasi-morphisms for the group $\Gamma$. It follows from our construction that for a suitable choice of the set $C$ the vector space $\Psi\left(\mathcal{H}_{C}\right)$ is infinite-dimensional. As in Section 2, the map $\Psi$ then induces via composition with the natural projection a linear map $\Theta: \mathcal{H}_{C} \rightarrow H_{b}^{2}(\Gamma, \mathbb{R})$. However, a priori the image of $\Theta$ may be trivial or finitedimensional. To establish that the subspace of $H_{b}^{2}(\Gamma, \mathbb{R})$ obtained in this way as the sets $C$ vary is infinite-dimensional, we use an additional assumption on $\Gamma$ which is motivated by the work [BF02] of Bestvina and Fujiwara. For this recall that a homeomorphism $g$ of $X$ which acts with north-south dynamics has an attracting fixed point $a \in X$ and a repelling fixed point $b \in X-\{a\}$. We call $(a, b)$ the ordered pair of fixed points for $g$. We show

Proposition 3.2. In the situation described in Lemma 3.1, assume in addition that the group $\Gamma$ contains a free subgroup $G$ with two generators and the following properties.

(1) Every $e \neq g \in G$ acts with north-south dynamics on $X$.

(2) There are infinitely many $g_{i} \in G(i>0)$ such that the $\Gamma$-orbits of the ordered pairs of fixed points of the elements $g_{i}, g_{j}^{-1}(i, j>0)$ are pairwise disjoint.

Then the images of the spaces $\mathcal{H}_{C}$ under the map $\Theta$ for suitable choices of $C \subset T$ span an infinite-dimensional subspace of $H_{b}^{2}(\Gamma, \mathbb{R})$.

Proof. Continue to use the assumptions and notations from Lemma 3.1 and its proof. We have to show that the bounded cohomology classes $\Theta(f)\left(f \in \mathcal{H}_{C}\right)$ defined by the quasi-morphisms $\Psi(f)$ constructed in Lemma 3.1 for suitable choices of $C$ span an infinite-dimensional subspace of the kernel of the map $H_{b}^{2}(\Gamma, \mathbb{R}) \rightarrow H^{2}(\Gamma, \mathbb{R})$. For this let $G$ be the free subgroup of $\Gamma$ with two generators as in the statement of the proposition. 
Let $g, h \in G-\{e\}$ be such that the $\Gamma$-orbit of the ordered pair $(a, b)$ of fixed points for $g$ is distinct from the $\Gamma$-orbit of the ordered pair $\left(a^{\prime}, b^{\prime}\right)$ of fixed points for $h$. Then the leaves $F(a, b), F\left(a^{\prime}, b^{\prime}\right)$ of the foliation $\mathcal{F}$ project to distinct leaves $L, L^{\prime}$ of the induced foliation $\mathcal{F}_{0}$ on $Y=T / \Gamma$. We claim that the closures of these leaves do not intersect. For this denote as before by $\pi: T \rightarrow Y$ the natural projection. Let $\epsilon_{0}>0$ be sufficiently small that $d\left(\{a, b\},\left\{a^{\prime}, b^{\prime}\right\}\right) \geq 2 \epsilon_{0}$. Since $g, h$ act on $X$ with north-south dynamics and fixed points $a, b$ and $a^{\prime}, b^{\prime}$, there is a number $\epsilon<\epsilon_{0}$ with the property that the projection $\pi$ maps the set $\{(a, b, x) \mid d(x,\{a, b\}) \geq \epsilon\}$ onto $L$ and that $\pi$ maps $\left\{\left(a^{\prime}, b^{\prime}, y\right) \mid d\left(y,\left\{a^{\prime}, b^{\prime}\right\}\right) \geq \epsilon\right\}$ onto $L^{\prime}$.

Assume to the contrary that the closures of the leaves $L, L^{\prime}$ contain a common point. By the above observation, this implies that there is a sequence $\left(g_{i}\right) \subset \Gamma$ of pairwise distinct elements and there are sequences $\left(x_{i}\right) \subset X,\left(y_{i}\right) \subset X$ such that

$$
d\left(x_{i},\{a, b\}\right) \geq \epsilon, \quad d\left(y_{i},\left\{a^{\prime}, b^{\prime}\right\}\right) \geq \epsilon \quad \text { for all } i
$$

and that $d\left(g_{i}\left(a, b, x_{i}\right),\left(a^{\prime}, b^{\prime}, y_{i}\right)\right) \rightarrow 0$. In particular, for every $\delta>0$ there are infinitely many distinct elements $u \in \Gamma$ such that $d\left(a^{\prime}, u a\right)<\delta, d\left(b^{\prime}, u b\right)<\delta$ and that $u(X-$ $B(a, \epsilon)-B(b, \epsilon)) \cap X-B\left(a^{\prime}, \epsilon\right)-B\left(b^{\prime}, \epsilon\right) \neq \emptyset$. However, this contradicts the second requirement in the definition of a metrically proper action. As a consequence, the closures of the leaves $L, L^{\prime}$ in $Y$ are disjoint.

Let $g \in G$ and let $a$ be the attracting and $b$ be the repelling fixed point of $g$. Choose the set $C=U \times V \times W \subset T$ as in Lemma 3.1 and its proof in such a way that $a \in U$ and $b \in V$. This is possible since the action of $\Gamma$ on $X$ is metrically proper and hence the stabilizer of $\{a, b\}$ in $\Gamma$ acts freely on an open subset of $X-\{a, b\}$. Let $x \in U-\{a\}$ and choose a closed neighborhood $A \subset U-\{a\}$ of $x$ for the construction of the quasimorphism $\Psi(f)$. Since $g$ acts on $X$ with north-south dynamics there is a closed subset $D$ of $X-\{a, b\}$ with dense interior whose distance to $\{a, b\}$ is positive and which is a fundamental domain for the action on $X-\{a, b\}$ of the cyclic subgroup of $G$ generated by $g$. For the measures $\mu_{\mathcal{F}}$ on the leaves of the foliation $\mathcal{F}$ as in the proof of Lemma 3.1 we may assume that the support of $\mu_{\mathcal{F}}$ intersects $F(a, b)$ and that the $\mu_{\mathcal{F}}$-mass of the boundary of $D$ viewed as a subset of $F(a, b)$ vanishes. Let $f \in \mathcal{H}_{C}$ and let $\tilde{f}$ be the $\Gamma$-invariant $\iota$-anti-invariant function on $T$ defined by $f$ as in the proof of Lemma 3.1. By the discussion in Step 1 of the proof of Lemma 3.1, the integral $\int_{D} \tilde{f} d \mu_{\mathcal{F}}$ exists. Let $\Psi(f)$ be the quasi-morphism of $\Gamma$ defined by $f$ as in (2) in the proof of Lemma 3.1. We claim that

$$
\lim _{k \rightarrow \infty} \Psi(f)\left(g^{k}\right) / k=\int_{D} \tilde{f} d \mu_{\mathcal{F}}
$$

To show the claim, observe that as $k \rightarrow \infty$ the diameter of the sets $g^{k} A$ tends to 0 and $g^{k} x \rightarrow a \in X-A$. Choose a small closed ball $B \subset V$ about $b$. By the proof of Lemma 3.1 , for sufficiently large $k$ the absolute value of the difference

$$
\int_{F\left(g^{k} x, b\right)-g^{k} A-B} \tilde{f} d \mu_{\mathcal{F}}-\int_{F\left(g^{k} x, x\right)-g^{k} A-A} \tilde{f} d \mu_{\mathcal{F}}
$$

is bounded from above by a constant not depending on $k$. As a consequence, it is enough 
to show that

$$
\int_{F\left(g^{k} x, b\right)-g^{k} A-B} \tilde{f} d \mu_{\mathcal{F}} / k \rightarrow \int_{D} \tilde{f} d \mu_{\mathcal{F}} \quad(k \rightarrow \infty),
$$

and this in turn is equivalent to

$$
\int_{F(x, b)-A-g^{-k} B} \tilde{f} d \mu_{\mathcal{F}} / k \rightarrow \int_{D} \tilde{f} d \mu_{\mathcal{F}} \quad(k \rightarrow \infty) .
$$

Choose in particular $B=\{b\} \cup \bigcup_{j \leq 0} g^{j} D$. Then $B-g^{-k} B=\bigcup_{j=0}^{k-1} g^{-j} D$ for every $k>0$. Thus for every small ball $E \subset X-B$ about the attracting fixed point $a$ for $g$ we have

$$
\lim _{k \rightarrow \infty} \int_{F(x, b)-A-g^{-k} B} \tilde{f} d \mu_{\mathcal{F}} / k=\lim _{k \rightarrow \infty} \int_{F(a, b)-E-g^{-k} B} \tilde{f} d \mu_{\mathcal{F}} / k=\int_{D} \tilde{f} d \mu_{\mathcal{F}}
$$

This shows the above claim.

Let again $g \in G$ with attracting fixed point $a \in X$, repelling fixed point $b \in X-\{a\}$ and assume that the ordered pair $(a, b)$ is not contained in the $\Gamma$-orbit of the ordered pair $\iota(a, b)=(b, a)$. By the above consideration, the closure of the projection of the leaf $F(a, b)$ to $Y$ is disjoint from the closure of the projection of $\iota F(a, b)=F(b, a)$. As before, let $D \subset F(a, b)$ be a closed fundamental domain for the action on $X-\{a, b\} \sim$ $F(a, b)$ of the cyclic group generated by $g$. By the second requirement in the definition of a metrically proper action, there are only finitely many $h \in \Gamma$ with $h D \cap D \neq \emptyset$. Denote by $\pi: T \rightarrow Y$ the canonical projection. The measures $\mu_{\mathcal{F}}$ project to a family of measures on the leaves of the foliation $\mathcal{F}_{0}=\pi \mathcal{F}$ on $Y$. For $f \in \mathcal{H}_{C}$ the function $\tilde{f}$ projects to a function $f_{0}$ on $Y$. Since $h D \cap D \neq \emptyset$ for only finitely many $h \in \Gamma$, the integral $\int_{D} \tilde{f} d \mu_{\mathcal{F}}$ is a positive bounded multiple of $\int_{\pi F(a, b)} f_{0} d \mu_{0}$. By the above considerations, the closure $L$ of the projection of the leaf $F(a, b)$ to $Y$ is disjoint from the closure of its image $F(b, a)$ under the involution $\iota$ and therefore for any given number $q \in \mathbb{R}$ there is a Hölder function $f \in \mathcal{H}_{C}$ such that the quasi-morphism $\Psi(f)$ defined as above by $f$ satisfies $\lim _{k \rightarrow \infty} \Psi(f)\left(g^{k}\right) / k=q$.

By our assumption, there are infinitely many elements $g_{i} \in G(i>0)$ which act on $X$ with north-south dynamics and such that the ordered pairs of fixed points of $g_{i}, g_{j}^{-1}$ are pairwise contained in distinct $\Gamma$-orbits on $X$. In particular, for $i \neq j$ the closures of the projections to $Y$ of the leaves of the foliation $\mathcal{F}$ which are determined by the fixed points of $g_{i}, g_{j}$ are disjoint. Now for any finite set $\left\{h_{1}, \ldots, h_{m}\right\} \subset\left\{g_{i} \mid i>0\right\} \subset G$ choose the set $C$ as above in such a way that it intersects each of the leaves of $\mathcal{F}$ determined by the ordered pair of fixed points of $h_{i}$; this can always be achieved by allowing for our construction a set $C$ which consists of finitely many components satisfying each of the above assumptions. The above discussion shows that for an arbitrarily chosen collection $\left\{q_{1}, \ldots, q_{m}\right\} \subset \mathbb{R}$ of real numbers there is a suitable choice of the function $f \in \mathcal{H}_{C}$ so that the quasi-morphism $\Psi(f)$ for $\Gamma$ defined by $f$ satisfies $\lim _{k \rightarrow \infty} \Psi(f)\left(h_{i}^{k}\right) / k=q_{i}$ for $1 \leq i \leq k$. 
For $f \in \mathcal{H}_{C}$ the cohomology class $\Theta(f) \in H_{b}^{2}(\Gamma, \mathbb{R})$ vanishes if and only if there is a homomorphism $\eta \in H^{1}(\Gamma, \mathbb{R})$ such that $\sup _{g \in \Gamma}|\Psi(f)(g)-\eta(g)|<\infty$ (cf. the discussion in Section 2). This homomorphism then restricts to a homomorphism of the group $G$. Now $G$ is a free group with two generators and hence $H^{1}(G, \mathbb{R})=\mathbb{R}^{2}$. More precisely, if $u_{1}, u_{2}$ are such free generators for $G$ then every homomorphism $\eta: G \rightarrow \mathbb{R}$ is determined by its value at $u_{1}, u_{2}$. In particular, for any finite subset $\left\{h_{1}, \ldots, h_{m}\right\} \subset G$ there are two elements in this collection, say $h_{1}, h_{2}$, such that for every quasi-morphism $\eta$ for $G$ which is equivalent to a homomorphism and every $j \in\{3, \ldots, m\}$ the quantity $\lim _{k \rightarrow \infty} \eta\left(h_{j}^{k}\right) / k$ is uniquely determined by $\lim _{k \rightarrow \infty} \eta\left(h_{i}^{k}\right) / k(i=1,2)$. Together with the above observation that for any finite subset $\left\{h_{1}, \ldots, h_{m}\right\}$ of $\left\{g_{i} \mid i>0\right\}$ we can find a quasi-morphism for $\Gamma$ for which these limits assume arbitrarily prescribed values we conclude that there are infinitely many quasi-morphisms for $\Gamma$ whose restrictions to $G$ define linearly independent elements of $H_{b}^{2}(G, \mathbb{R})$. This shows that the kernel of the map $H_{b}^{2}(\Gamma, \mathbb{R}) \rightarrow H^{2}(\Gamma, \mathbb{R})$ is infinite-dimensional and completes the proof of the proposition.

Remark. The above proof also shows the following. Let $\Gamma$ be a countable group which admits a weakly hyperbolic action by homeomorphisms of a metric space $X$ of finite diameter such that the action of $\Gamma$ on $T=X^{3}-\Delta$ is metrically proper. Let $g_{i} \in$ $\Gamma$ be elements which act with north-south dynamics on $X$ with ordered pairs of fixed points $\left(a_{i}, b_{i}\right)(i=1, \ldots, k)$. If the $\Gamma$-orbits of $\left(a_{i}, b_{i}\right),\left(b_{j}, a_{j}\right)(i, j \leq k)$ are all disjoint then for every tuple $\left(q_{1}, \ldots, q_{k}\right) \in \mathbb{R}^{k}$ there is a quasi-morphism $\varphi$ for $\Gamma$ with $\lim _{l \rightarrow \infty} \varphi\left(g_{i}^{l}\right) / l=q_{i}$ for every $i \leq k$.

The following theorem is the main technical result of this note. For its formulation, recall that the free group $G$ with two generators is the fundamental group of a convex cocompact hyperbolic surface whose limit set $B$ is just the Gromov boundary of $G$.

Theorem 3.3. Let $(X, d)$ be a metric space of finite diameter without isolated points. Let $\Gamma$ be a countable group which admits a weakly hyperbolic action by homeomorphisms of $X$. Assume that $\Gamma$ contains a free subgroup $G$ with two generators and the following properties.

(1) Every $e \neq g \in G$ acts with north-south dynamics on $X$.

(2) There are infinitely many $g_{i} \in G(i>0)$ such that the $\Gamma$-orbits of the ordered pairs of fixed points of the elements $g_{i}, g_{j}^{-1}(i, j>0)$ are pairwise disjoint.

(3) There is a G-equivariant continuous embedding of the Gromov boundary of G into X.

If the action of $\Gamma$ on the space of triples of pairwise distinct points in $X$ is metrically proper then for every $p \in(1, \infty)$ the kernel of the map $H_{b}^{2}\left(\Gamma, \ell^{p}(\Gamma)\right) \rightarrow H^{2}\left(\Gamma, \ell^{p}(\Gamma)\right)$ is infinite-dimensional.

Proof. Let $\Gamma$ be a countable group acting by homeomorphisms on a metric space $(X, d)$ of finite diameter without isolated points. Assume that the action of $\Gamma$ is weakly hyperbolic and that the diagonal action of $\Gamma$ on the space $T=X^{3}-\Delta$ of triples of pairwise distinct points in $X$ is metrically proper. Write $Y=T / \Gamma$ and denote as before by 
$\iota: T \rightarrow T$ the natural involution which exchanges the first two points in a triple. Let $G$ be a free subgroup of $\Gamma$ with two generators as in the statement of the theorem. In particular, we assume that there is a continuous $G$-equivariant embedding of the Gromov boundary $B$ of $G$ into $X$. We have to show that for every $p \in(1, \infty)$ the kernel of the map $H_{b}^{2}\left(\Gamma, \ell^{p}(\Gamma)\right) \rightarrow H^{2}\left(\Gamma, \ell^{p}(\Gamma)\right)$ is infinite-dimensional.

Denote by \|\|$_{p}$ the norm of the Banach space $\ell^{p}(\Gamma)$. We assume that $\Gamma$ acts on $\ell^{p}(\Gamma)$ by right translation, i.e. for every $g \in \Gamma$ and every function $\psi \in \ell^{p}(\Gamma)$ we have $(g \psi)(h)=\psi(h g)$. Define an $\ell^{p}(\Gamma)$-valued quasi-morphism for $\Gamma$ to be a map $\eta: \Gamma \rightarrow \ell^{p}(\Gamma)$ such that

$$
\sup _{g, h \in \Gamma}\|\eta(g)+g \eta(h)-\eta(g h)\|_{p}<\infty .
$$

Two such quasi-morphisms $\eta, \eta^{\prime}$ are called equivalent if $\eta-\eta^{\prime}$ is bounded as a function from $\Gamma$ to $\ell^{p}(\Gamma)$, i.e. if there is a number $c>0$ such that $\left\|\left(\eta-\eta^{\prime}\right)(g)\right\|_{p} \leq c$ for all $g \in \Gamma$.

By Corollary 7.4.7 in [M01], the cohomology group $H_{b}^{2}\left(\Gamma, \ell^{p}(\Gamma)\right)$ coincides with the second cohomology group of the complex

$$
0 \rightarrow L^{\infty}\left(\Gamma, \ell^{p}(\Gamma)\right)^{\Gamma} \stackrel{d}{\rightarrow} L^{\infty}\left(\Gamma^{2}, \ell^{p}(\Gamma)\right)^{\Gamma} \stackrel{d}{\rightarrow} L^{\infty}\left(\Gamma^{3}, \ell^{p}(\Gamma)\right)^{\Gamma} \rightarrow \cdots
$$

with the usual homogeneous coboundary operator $d$. Let $\psi: \Gamma^{2} \rightarrow \ell^{p}(\Gamma)$ be any (unbounded) $\Gamma$-equivariant map; this means that $\psi\left(h g_{1}, h g_{2}\right)=h\left(\psi\left(g_{1}, g_{2}\right)\right)$ for all $g_{1}, g_{2}, h \in \Gamma$. If the image $d \psi$ of $\psi$ under the coboundary operator $d$ is bounded, then as in the case of real coefficients, the map $\psi$ defines a class in the kernel of the natural map $H_{b}^{2}\left(\Gamma, \ell^{p}(\Gamma)\right) \rightarrow H^{2}\left(\Gamma, \ell^{p}(\Gamma)\right)$. Let $e$ be the unit element in $\Gamma$ and define a map $\varphi: \Gamma \rightarrow \ell^{p}(\Gamma)$ by $\varphi(v)=\psi(e, v)$. Then for $g, h, u \in \Gamma$ we have $d \psi(g, h, u)=$ $\psi(h, u)-\psi(g, u)+\psi(g, h)=h \varphi\left(h^{-1} u\right)-g \varphi\left(g^{-1} u\right)+g \varphi\left(g^{-1} h\right)=g\left(\varphi\left(g^{-1} h\right)+\right.$ $\left.g^{-1} h \varphi\left(h^{-1} u\right)-\varphi\left(g^{-1} u\right)\right)$. Since $\Gamma$ acts isometrically on $\ell^{p}(\Gamma)$, we conclude that $d \psi$ is bounded if and only if $\varphi$ defines an $\ell^{p}(\Gamma)$-valued quasi-morphism for $\Gamma$. Now by equivariance, $\psi$ is uniquely determined by $\varphi$ and therefore every equivalence class of an $\ell^{p}(\Gamma)$ valued quasi-morphism gives rise to a cohomology class in the kernel of the natural map $H_{b}^{2}\left(\Gamma, \ell^{p}(\Gamma)\right) \rightarrow H^{2}\left(\Gamma, \ell^{p}(\Gamma)\right)$. This cohomology class vanishes if and only if there is a map $\eta: \Gamma \rightarrow \ell^{p}(\Gamma)$ which satisfies $\eta(g h)=\eta(g)+g \eta(h)$ for all $g, h \in \Gamma$ and such that $\varphi-\eta$ is bounded.

Let again $T$ be the space of triples of pairwise distinct points in $X$. The group $\Gamma$ and the involution $\iota$ act on $T$, and these actions commute; we denote as before by $Z$ the corresponding quotient. As above, let $C \subset T$ be a set of positive distance to $\Delta$ and sufficiently small diameter which is mapped homeomorphically into the quotient $Z$.

Let $\hat{T}=T \times \Gamma$ and define $\hat{\mathcal{H}}$ to be the vector space of all functions $f: \hat{T} \rightarrow \mathbb{R}$ supported in $C \times \Gamma$ with the following property. For $g \in \Gamma$ write $f_{g}(x)=f(x, g)$; we view $f_{g}$ as a function $C \rightarrow \mathbb{R}$. We require that there is some $\alpha \in(0,1)$ such that the Hölder- $\alpha$-norms $\left\|f_{g}\right\|_{\alpha}$ of the functions $f_{g}(g \in \Gamma)$ on $C$ satisfy $\sum_{g \in \Gamma}\left\|f_{g}\right\|_{\alpha}^{p}<\infty$. Then for each $y \in C$ the function $f_{y}: g \mapsto f(y, g)$ is contained in $\ell^{p}(\Gamma)$ and therefore the assignment $y \in C \mapsto f_{y}$ defines a (Hölder continuous) map of $C$ into $\ell^{p}(\Gamma)$. The set of all such functions naturally has the structure of an infinite-dimensional vector 
space. Extend the function $f \in \hat{\mathcal{H}}$ to a function $\hat{f}$ on $\hat{T}$ which is anti-invariant under the involution $\iota:(\zeta, g)=(\iota \zeta, g)$ and satisfies $\hat{f}(g z, u)=f(z, u g)$ for all $z \in T$ and all $g, u \in \Gamma$.

As in the proof of Lemma 3.1 above, assume that $C=U \times V \times W$ for open subsets $U, V, W$ of positive distance and sufficiently small diameter. Recall from the proof of Lemma 3.1 the definition of the foliation $\mathcal{F}$ of $T$ and the measures $\mu_{\mathcal{F}}$. Choose a small closed ball $A \subset U$, a point $x \in A$ and for $g \in \Gamma$ define a function $\Psi(f)(g): \Gamma \rightarrow \mathbb{R}$ by

$$
\Psi(f)(g)(u)=\int_{F(x, g x)-A-g A} \hat{f}(y, u) d \mu_{\mathcal{F}}(y) .
$$

It follows from the choice of $f$ and the considerations in Step 1 of the proof of Lemma 3.1 that $\Psi(f)(g) \in \ell^{p}(\Gamma)$. On the other hand, by the definition of the function $\hat{f}$ we have

$$
\begin{aligned}
\int_{F(g x, g h x)-g A-g h A} \hat{f}(y, u) d \mu_{\mathcal{F}}(y) & =\int_{F(x, h x)-A-h A} \hat{f}(y, u g) d \mu_{\mathcal{F}}(y) \\
& =\Psi(f)(h)(u g)
\end{aligned}
$$

and consequently the estimates in Step 2 of the proof of Lemma 3.1 show that the map $\Psi(f)$ is an $\ell^{p}(\Gamma)$-valued quasi-morphism for $\Gamma$. In other words, as in the case of real coefficients we obtain a linear map $\Theta$ from the vector space $\hat{\mathcal{H}}$ into the kernel of the natural map $H_{b}^{2}\left(\Gamma, \ell^{p}(\Gamma)\right) \rightarrow H^{2}\left(\Gamma, \ell^{p}(\Gamma)\right)$ which assigns to a function $f \in \hat{\mathcal{H}}$ the cohomology class of the $\ell^{p}(\Gamma)$-valued quasi-morphism $\Psi(f)$.

Our goal is to show that the image of the map $\Theta$ is infinite-dimensional. For this let $G<\Gamma$ be the free group with two generators as in the statement of the theorem. Then every function $u \in \ell^{p}(\Gamma)$ restricts to a function $R u \in \ell^{p}(G)$, and for $g \in G$ we have $R(g u)=g(R u)$. Thus for every $f \in \hat{\mathcal{H}}$ the map $\Psi(f): \Gamma \rightarrow \ell^{p}(\Gamma)$ restricts to an $\ell^{p}(G)$-valued quasi-morphism $R \Psi(f): G \rightarrow \ell^{p}(G)$ which defines a cohomology class $R \Theta(f) \in H_{b}^{2}\left(G, \ell^{p}(G)\right)$. If the cohomology class $\Theta(f)$ vanishes then the same is true for the cohomology class $R \Theta(f)$. Thus it is enough to show that the subspace $\{R \Theta(f) \mid f \in \hat{\mathcal{H}}\}$ of $H_{b}^{2}\left(G, \ell^{p}(G)\right)$ is infinite-dimensional.

For this let $B$ be the Gromov boundary of the free group $G$; this boundary is a Cantor set on which the group $G$ acts as a group of homeomorphisms with north-south dynamics. Assume that there is a $G$-equivariant continuous embedding $\rho_{0}: B \rightarrow X$. If we denote by $B T$ the space of triples of pairwise distinct points in $B$ then the map $\rho_{0}$ induces a continuous $G$-equivariant embedding $\rho: B T \rightarrow T$. In the following we identify $B T$ with its image under $\rho$, i.e. we suppress the map $\rho$ in our notations. Let $f \in \hat{\mathcal{H}}$; for a triple $\left(x_{1}, x_{2}, x_{3}\right) \in B T$ and $u \in G$ define

$$
\begin{aligned}
v(f)\left(x_{1}, x_{2}, x_{3}\right)(u)= & \int_{F\left(x_{1}, x_{2}\right)} \hat{f}(y, u) d \mu_{\mathcal{F}}(y)+\int_{F\left(x_{2}, x_{3}\right)} \hat{f}(y, u) d \mu_{\mathcal{F}}(y) \\
& +\int_{F\left(x_{3}, x_{1}\right)} \hat{f}(y, u) d \mu_{\mathcal{F}}(y) .
\end{aligned}
$$

Here the sum on the right hand side of the equation is viewed as a limit of sums of finite integrals over the complements in the leaves $F\left(x_{i}, x_{j}\right)$ of smaller and smaller neighborhoods of the points $x_{i}(i=1,2,3)$, and its existence follows as above from the con- 
tinuity properties of the function $\hat{f}$. By the choice of $f$, for every $\left(x_{1}, x_{2}, x_{3}\right) \in B T$ the function $u \in G \mapsto v(f)\left(x_{1}, x_{2}, x_{3}\right)(u)$ is contained in $\ell^{p}(G)$. More precisely, the map $\left(x_{1}, x_{2}, x_{3}\right) \in B T \mapsto v(f)\left(x_{1}, x_{2}, x_{3}\right) \in \ell^{p}(\Gamma)$ is a continuous cocycle for the action of $G$ on $B$, i.e. it is continuous and equivariant under the action of $G$, it satisfies $\nu \circ \sigma=(\operatorname{sgn}(\sigma)) v$ for every permutation $\sigma$ of the three variables and the cocycle identity

$$
v(f)\left(x_{2}, x_{3}, x_{4}\right)-v(f)\left(x_{1}, x_{3}, x_{4}\right)+v(f)\left(x_{1}, x_{2}, x_{4}\right)-v(f)\left(x_{1}, x_{2}, x_{3}\right)=0 .
$$

In particular, for any fixed point $x \in B$ we conclude as in Section 2 that the assignment $\left(g_{1}, g_{2}, g_{3}\right) \mapsto v(f)\left(g_{1} x, g_{2} x, g_{3} x\right)\left(g_{i} \in G\right)$ defines a $G$-equivariant cocycle with values in $\ell^{p}(G)$ whose cohomology class coincides with $R \Theta(f)$.

Now by a result of Adams [A94] (see also [Ka03] for a more precise result), if $\sigma$ is the measure class of the measure of maximal entropy for the geodesic flow of any convex cocompact hyperbolic manifold whose fundamental group is a free group with two generators, viewed as a $G$-invariant measure class on the Gromov boundary $B$ of $G$, then $(B, \sigma)$ is a strong boundary for $G$. This means that the action of $G$ on $(B, \sigma)$ is amenable and doubly ergodic with respect to any separable Banach coefficient module, i.e. for every separable Banach $G$-space $E$, every measurable $G$-equivariant map $(B \times$ $B, \sigma \times \sigma) \rightarrow E$ is constant almost everywhere. As a consequence, every continuous $G$-equivariant cocycle $B T \rightarrow \ell^{p}(G)$ which does not vanish identically defines a nonvanishing class in $H_{b}^{2}\left(G, \ell^{p}(G)\right.$ ) (see the discussion in Section 7 of [M01]). Thus for every $f \in \hat{\mathcal{H}}$ such that $v(f) \neq 0$ the class $R \Theta(f)$ does not vanish and hence the same is true for the class $\Theta(f)$. In other words, to show that indeed $H_{b}^{2}\left(\Gamma, \ell^{p}(\Gamma)\right)$ is infinitedimensional we only have to find for every $m>0$ a collection of functions $f_{i} \in \hat{\mathcal{H}}$ $(1 \leq i \leq m)$ such that the cocycles $v\left(f_{i}\right)$ are linearly independent.

For this recall that by Proposition 3.2 and its proof, the subspace of $H_{b}^{2}(G, \mathbb{R})$ defined by the cohomology classes $\Theta_{G}(f) \in H_{b}^{2}(G, \mathbb{R})$ of the quasi-morphisms $\Psi(\alpha)$ where $\alpha \in \mathcal{H}_{C}$ for a suitable choice of $C \subset T$ is infinite-dimensional (note that we use here the notations from Lemma 3.1 for the map $\Psi$ ). On the other hand, for every $\alpha \in \mathcal{H}_{C}$ the cohomology class $\Theta_{G}(\alpha) \in H_{b}^{2}(G, \mathbb{R})$ coincides with the class defined by the continuous $\mathbb{R}$-valued cocycle $\nu_{0}(\alpha): B T \rightarrow \mathbb{R}$ given by

$$
\begin{aligned}
\nu_{0}(\alpha)\left(x_{1}, x_{2}, x_{3}\right)= & \int_{F\left(x_{1}, x_{2}\right)} \tilde{\alpha}(y) d \mu_{\mathcal{F}}(y)+\int_{F\left(x_{2}, x_{3}\right)} \tilde{\alpha}(y) d \mu_{\mathcal{F}}(y) \\
& +\int_{F\left(x_{3}, x_{1}\right)} \tilde{\alpha}(y) d \mu_{\mathcal{F}}(y) .
\end{aligned}
$$

Now let $C \subset T$ and let $\alpha_{1}, \ldots, \alpha_{m} \in \mathcal{H}_{C}$ be such that the cocycles $v_{0}\left(\alpha_{i}\right)$ are linearly independent; such functions exist by Proposition 3.2 and its proof. For every $i \leq m$ define a function $f_{i} \in \hat{\mathcal{H}}$ by $f_{i}(y, e)=\alpha_{i}(y)$ and $f_{i}(y, g) \equiv 0$ for $g \neq e$. Then

$$
\begin{aligned}
v_{0}\left(\alpha_{i}\right)\left(x_{1}, x_{2}, x_{3}\right)= & \int_{F\left(x_{1}, x_{2}\right)} \sum_{u \in G} \hat{f}_{i}(y, u) d \mu_{\mathcal{F}}(y)+\int_{F\left(x_{2}, x_{3}\right)} \sum_{u \in G} \hat{f}_{i}(y, u) d \mu_{\mathcal{F}}(y) \\
& +\int_{F\left(x_{3}, x_{1}\right)} \sum_{u \in G} \hat{f}_{i}(y, u) d \mu_{\mathcal{F}}(y)
\end{aligned}
$$


and therefore since the cocycles $\nu_{0}\left(\alpha_{i}\right)$ are linearly independent the same is true for the cocycles $v\left(f_{i}\right)$. As a consequence, the kernel of the map $H_{b}^{2}\left(\Gamma, \ell^{p}(\Gamma)\right) \rightarrow H^{2}\left(\Gamma, \ell^{p}(\Gamma)\right)$ is indeed infinite-dimensional.

\section{Groups acting isometrically on hyperbolic geodesic metric spaces}

In this section we consider countable groups which admit a weakly acylindrical isometric action on an arbitrary Gromov hyperbolic geodesic metric space $X$. We show that the assumptions in Theorem 3.3 are satisfied for the action of such a group $\Gamma$ on the Gromov boundary $\partial X$ of $X$. From this we deduce Theorem A from the introduction.

First recall that the Gromov boundary of a hyperbolic geodesic metric space $X$ is defined as follows. For a fixed point $x_{0} \in X$, define the Gromov product $(y, z)_{x_{0}}$ based at $x_{0}$ of two points $y, z \in X$ by

$$
(y, z)_{x_{0}}=\frac{1}{2}\left(d\left(y, x_{0}\right)+d\left(z, x_{0}\right)-d(y, z)\right) .
$$

Call two sequences $\left(y_{i}\right),\left(z_{j}\right) \subset X$ equivalent if $\left(y_{i}, z_{i}\right)_{x_{0}} \rightarrow \infty(i \rightarrow \infty)$. By hyperbolicity of $X$, this notion of equivalence defines an equivalence relation in the collection of all sequences $\left(y_{i}\right) \subset X$ with the additional property that $\left(y_{i}, y_{j}\right)_{x_{0}} \rightarrow \infty(i, j \rightarrow \infty)$ [BH99]. The boundary $\partial X$ of $X$ is the set of equivalence classes of this relation.

The Gromov product $(,)_{x_{0}}$ for pairs of points in $X$ can be extended to a product on $\partial X$ by defining

$$
(\xi, \eta)_{x_{0}}=\sup \liminf _{i, j \rightarrow \infty}\left(y_{i}, z_{j}\right)_{x_{0}}
$$

where the supremum is taken over all sequences $\left(y_{i}\right),\left(z_{j}\right) \subset X$ whose equivalence classes define the points $\xi, \eta \in \partial X$. For a suitable number $\chi>0$ only depending on the hyperbolicity constant of $X$ there is a distance $\delta=\delta_{x_{0}}$ of bounded diameter on $\partial X$ with the property that the distance $\delta(\xi, \eta)$ between two points $\xi, \eta \in \partial X$ is comparable to $e^{-\chi(\xi, \eta)_{x_{0}}}$ (see 7.3 of [GH90]). More precisely, there is a constant $\theta>0$ such that

$$
e^{-\chi \theta} e^{-\chi(\xi, \eta)_{x_{0}}} \leq \delta(\xi, \eta) \leq e^{-\chi(\xi, \eta)_{x_{0}}}
$$

for all $\xi, \eta \in \partial X$. In the following we always assume that $\partial X$ is equipped with such a distance $\delta$.

There is a natural topology on $X \cup \partial X$ which restricts to the given topology on $X$ and to the topology on $\partial X$ induced by the metric $\delta$. With respect to this topology, a sequence $\left(y_{i}\right) \subset X$ converges to $\xi \in \partial X$ if and only if we have $\left(y_{i}, y_{j}\right)_{x_{0}} \rightarrow \infty$ and the equivalence class of $\left(y_{i}\right)$ defines $\xi$. If $X$ is proper, then $X \cup \partial X$ is compact. Every isometry of $X$ acts naturally on $X \cup \partial X$ as a homeomorphism. We denote by $\operatorname{Iso}(X)$ the isometry group of $X$.

Since we do not assume that $X$ is proper, for a given pair of distinct points $\xi \neq \eta \in \partial X$ there may not exist a geodesic $\gamma$ in $X$ connecting $\xi$ to $\eta$, i.e. such that $\gamma(t)$ converges to $\xi$ as $t \rightarrow-\infty$ and that $\gamma(t)$ converges to $\eta$ as $t \rightarrow \infty$. However, there is a number $L>1$ only depending on the hyperbolicity constant for $X$ such that any two points in $\partial X$ can be connected by an $L$-quasi-geodesic. Recall that for $L \geq 1$, an $L$-quasi-geodesic in $X$ is 
a map $\gamma:(a, b) \rightarrow X$ for $-\infty \leq a<b \leq \infty$ such that

$$
-L+|s-t| / L \leq d(\gamma(s), \gamma(t)) \leq L|s-t|+L
$$

for all $s, t \in(a, b)$. Note that an $L$-quasi-geodesic $\gamma$ need not be continuous. However, from every $L$-quasi-geodesic $\gamma$ we can construct a continuous $4 L$-quasi-geodesic $\tilde{\gamma}$ whose Hausdorff distance to $\gamma$ is bounded from above by $4 L$ by replacing for each $i \geq 0$ the arc $\gamma[i, i+1]$ by a geodesic arc $\tilde{\gamma}[i, i+1]$ with the same endpoints. In other words, via changing the constant $L$ we may assume that for any two distinct points $\xi \neq \eta \in \partial X$ there is a continuous $L$-quasi-geodesic $\gamma$ connecting $\xi$ to $\eta$; we then write $\gamma(-\infty)=\xi, \gamma(\infty)=\eta$ (see [GH90, 5.25 and 7.6]; cf. also the discussion in [H06]).

Recall from Section 3 the definition of a weakly hyperbolic action of a group $G$ on a metric space of bounded diameter. We show

Lemma 4.1. Let $X$ be an arbitrary hyperbolic geodesic metric space. Then the action of the isometry group Iso $(X)$ on $\partial X$ is weakly hyperbolic.

Proof. The boundary $\partial X$ of a hyperbolic geodesic metric space $X$ is a metric space of bounded diameter where the metric $\delta$ is constructed from the Gromov product $(,)_{x_{0}}$ at a fixed point $x_{0} \in X$. There are numbers $\chi, \theta>0$ such that inequality (8) above holds for the distance $\delta$.

Our goal is to show that for every $v>0$ there is a constant $\Theta=\Theta(v)>0$ with the following property. Let $a, b \in \partial X$ with $\delta(a, b) \geq 2 v$. Let $g \in \operatorname{Iso}(X)$ be such that $\delta(g a, g b) \geq 2 v$; if $v \in \partial X-\{a, b\}$ is such that $\min \{\delta(g v, g a), \delta(g v, g b)\} \geq v$ then $\delta(g w, g b) \leq \Theta \delta(v, a)$ for every $w \in \partial X$ with $\delta(w, b) \leq v$. Note that since the diameter of $\partial X$ is finite, this inequality is automatically satisfied for a suitable choice of $\Theta$ whenever $\delta(v, a)$ is bounded from below by a universal constant. Thus it is enough to show the claim under the additional assumption that $\delta(v, a) \leq \epsilon$ for some fixed $\epsilon>0$ which will be determined later on.

Let $T \subset(\partial X)^{3}$ be the set of all triples of pairwise distinct points in $\partial X$. A triple $(a, b, c) \in T$ determines (non-uniquely) an ideal $L$-quasi-geodesic triangle with vertices $a, b, c$. The Hausdorff distance between any two such $L$-quasi-geodesic triangles with the same vertices in $\partial X$ is bounded by a universal constant. There is a number $p_{0}>0$ such that for every $p \geq p_{0}$ and every triple $(a, b, c) \in T$ the closed set $K(a, b, c ; p) \subset X$ of all points in $X$ whose distance to each side of an $L$-quasi-geodesic triangle with vertices $a, b, c$ is at most $p$ is non-empty. The diameter of this set is uniformly bounded by a constant only depending on $p$ and the hyperbolicity constant for $X$.

By the definition of the Gromov product and hyperbolicity, there is a number $m_{1}>0$ with the following property. Let $(a, b, c) \in T$ and let $\zeta$ be a continuous $L$-quasi-geodesic connecting $b$ to $a$. Then $\min \left\{(a, c)_{\zeta(0)},(b, c)_{\zeta(0)}\right\} \leq m_{1}$ and if $(b, c)_{\zeta(0)} \leq(a, c)_{\zeta(0)}$ then we have $\zeta(\tau) \in K\left(a, b, c ; m_{1}\right)$ for every $\tau \geq 0$ such that $d(\zeta(0), \zeta(\tau))=(a, c)_{\zeta(0)}$.

Now let $v \in(0,1)$ and let $a, b \in \partial X$ be such that $\delta(a, b) \geq 2 v$. By hyperbolicity and inequality (8) above, there is a constant $m_{0}=m_{0}(v)>0$ such that every continuous $L$-quasi-geodesic connecting two points $a \neq b \in \partial X$ with $\delta(a, b) \geq v$ intersects the ball $B\left(x_{0}, m_{0}\right)$. Let $\gamma$ be a continuous $L$-quasi-geodesic connecting $b=\gamma(-\infty)$ to 
$a=\gamma(\infty)$ which is parametrized in such a way that $\gamma(0) \in B\left(x_{0}, m_{0}\right)$. Let $\theta, \chi>0$ be as in inequality (8), let $R_{0}=\chi\left(m_{0}+m_{1}+\theta\right)$ and let $v \in \partial X-\{a, b\}$ be such that $\delta(a, v) \leq e^{-R_{0}}$; then $\delta(a, v)=e^{-R}$ for some $R \geq R_{0}$. By inequality 8 , we have $R / \chi-\theta \leq(a, v)_{x_{0}} \leq R / \chi$ and hence

$$
R / \chi-\theta-m_{0} \leq(a, v)_{\gamma(0)} \leq R / \chi+m_{0}
$$

since $d\left(x_{0}, \gamma(0)\right) \leq m_{0}$. From the assumption on $R$ we find that $(a, v)_{\gamma(0)} \geq m_{1}$ and hence $\gamma(\tau) \in K\left(a, b, v ; m_{1}\right)$ for all $\tau \geq 0$ such that $d(\gamma(0), \gamma(\tau))=(a, v)_{\gamma(0)}$.

Let $g \in \operatorname{Iso}(X)$ be such that $\delta(g a, g b) \geq 2 v$ and $\min \{\delta(g a, g v), \delta(g b, g v)\} \geq v$. Then the $L$-quasi-geodesic $g \gamma$ intersects $B\left(x_{0}, m_{0}\right)$ and the same if true for any $L$ quasi-geodesic connecting $g a$ to $g v$ or connecting $g b$ to $g v$ and consequently $x_{0} \in$ $K\left(g a, g b, g v ; m_{0}\right)$. If as before $\tau>0$ is such that $d(\gamma(0), \gamma(\tau))=(a, v)_{\gamma(0)}$ then $\gamma(\tau) \in K\left(a, b, v ; m_{1}\right)$ and therefore

$$
\left\{x_{0}, g \gamma(\tau)\right\} \subset K\left(g a, g b, g v ; m_{0}+m_{1}\right)=g K\left(a, b, v ; m_{0}+m_{1}\right) .
$$

Now the diameter of the set $K\left(g a, g b, g v ; m_{0}+m_{1}\right)$ is bounded from above by a constant $m_{2}=m_{2}(v)>0$ only depending on $v$ and hence $d\left(g \gamma(\tau), x_{0}\right) \leq m_{2}$.

Let $w \in \partial X$ be such that $\delta(w, b) \leq v$. Then $\delta(w, a) \geq v$ and by inequality $(8)$ above, the Gromov product $(w, a)_{x_{0}}$ is bounded from above by a universal constant and the same is true for $(w, a)_{\gamma(0)}$. In particular, the $L$-quasi-geodesic ray $\gamma[0, \infty)$ connecting $\gamma(0)$ to $a$ is contained in a uniformly bounded neighborhood of any $L$-quasi-geodesic connecting $w$ to $a$. With $\tau>0$ as above we have $|d(\gamma(\tau), \gamma(0))-R / \chi| \leq m_{0}+\theta$ and hence by the definition of the Gromov product and hyperbolicity, the quantity $(b, w)_{\gamma(\tau)}-R / \chi=$ $(g b, g w)_{g \gamma(\tau)}-R / \chi$ is bounded from below by a universal constant. But $d\left(g \gamma(\tau), x_{0}\right) \leq$ $m_{2}$ and hence we have

$$
\left|(g b, g w)_{g \gamma(\tau)}-(g b, g w)_{x_{0}}\right|=\left|(b, w)_{\gamma(\tau)}-(g b, g w)_{x_{0}}\right| \leq m_{2} .
$$

Using once more the estimate $(8)$ we conclude that there is a number $\Theta>1$ only depending on $v$ such that $\delta(g b, g w) \leq \Theta e^{-R}=\Theta \delta(a, v)$. This shows that the action of $\operatorname{Iso}(X)$ on $\partial X$ is weakly hyperbolic.

As in the introduction, we call an isometric action on $X$ of a countable group $\Gamma$ weakly acylindrical if for every point $x_{0} \in X$ and every $m>0$ there are numbers $R\left(x_{0}, m\right)>0$ and $c\left(x_{0}, m\right)>0$ with the following property. If $x, y \in X$ with $d(x, y) \geq R\left(x_{0}, m\right)$ are such that a geodesic $\gamma$ connecting $x$ to $y$ meets the $m$-neighborhood of $x_{0}$ then there are at most $c\left(x_{0}, m\right)$ elements $g \in \Gamma$ such that $d(x, g x) \leq m$ and $d(y, g y) \leq m$. We have.

Lemma 4.2. Let $X$ be a hyperbolic geodesic metric space and let $\Gamma$ be a countable subgroup of $\operatorname{Iso}(X)$ whose action on $X$ is weakly acylindrical. Then the action of $\Gamma$ on the space or triples of pairwise distinct points in $\partial X$ is metrically proper.

Proof. The group $\Gamma$ acts as a group of homeomorphisms on the Gromov boundary $\partial X$ of $X$. Recall that $\partial X$ is a metric space of bounded diameter where the metric $\delta$ is constructed from the Gromov product $(,)_{x_{0}}$ at a fixed point $x_{0} \in X$ and it satisfies the 
estimate (8) from the beginning of this section for some $\chi, \theta>0$ and all $\xi \neq \eta \in \partial X$. We have to show that the action of $\Gamma$ on the space $T$ of triples of pairwise distinct points in $\partial X$ is metrically proper.

For this let $v>0$ be fixed. There are numbers $L \geq 1$ and $m_{0}=m_{0}(v)>0$ such that any two points $x \neq y \in \partial X$ can be connected by a continuous $L$-quasi-geodesic, and if $\delta(x, y) \geq v$ then this quasi-geodesic intersects the ball $B\left(x_{0}, m_{0}\right)$.

By hyperbolicity, the Hausdorff distance between any two $L$-quasi-geodesics connecting the same points in $\partial X$ is bounded from above by a universal constant. Moreover, there is a universal constant $m_{1}=m_{1}(v)>m_{0}$ with the following property. Let $a \neq b$, $x \neq y \in \partial X$ and assume that $\delta(a, b) \geq 2 v$ and that for some $R>-\log (v / 2)$ we have $\delta(a, x) \leq e^{-R}, \delta(b, y) \leq e^{-R}$. Let $\gamma$ be a continuous $L$-quasi-geodesic connecting $b=\gamma(-\infty)$ to $a=\gamma(\infty)$ and let $\eta$ be a continuous $L$-quasi-geodesic connecting $y=\eta(-\infty)$ to $x=\eta(\infty)$; then $\gamma, \eta$ intersect the ball $B\left(x_{0}, m_{0}\right)$, and the intersection of $\gamma$ with $B\left(x_{0}, R / \chi\right)$ is contained in the $m_{1}$-neighborhood of $\eta$.

As in the proof of Lemma 4.1, for $p>0$ and a triple $(u, v, w) \in T$ let $K(u, v, w ; p)$ $\subset X$ be the set of all points whose distance to each side of an $L$-quasi-geodesic triangle with vertices $u, v, w$ is at most $p$. By the arguments in the proof of Lemma 4.1 there is a constant $m_{2}>m_{1}$ with the following property. Let $x, y \in \partial X$ with $\delta(x, y) \geq v$. If $z \in \partial X$ and $k \geq-\log (v / 2)$ are such that $e^{-k} \leq \delta(x, z) \leq e^{-k+1}$ then the distance between $x_{0}$ and $K\left(x, y, z ; m_{0}\right)$ is contained in the interval $\left[k / \chi-m_{2}, k / \chi+m_{2}\right]$. The diameter of the sets $K\left(x^{\prime}, y^{\prime}, z^{\prime} ; m_{0}\right)$ is bounded from above by a universal constant $\rho>0$ only depending on $m_{0}$ and the hyperbolicity constant of $X$.

Let $(a, b, c) \in(\partial X)^{3}$ be a triple of points whose pairwise distances are at least $2 v$. Let $R \geq-\log (v / 2)$ be a number to be determined later, let $U(a), U(b), U(c)$ be the open $e^{-\bar{R}}$-neighborhood of $a, b, c$ in $\partial X$ and let $x \in U(a), y \in U(b), z \in U(c)$. Let $a^{\prime}, b^{\prime} \in$ $\partial X$ be such that $\delta\left(a^{\prime}, b^{\prime}\right) \geq 2 v$ and assume that there is some $g \in \Gamma$ such that $g x=a^{\prime}$, $g y=b^{\prime}$ and $\delta\left(g z, a^{\prime}\right) \in\left[e^{-k}, e^{-k+1}\right]$ for some $k \geq R$. Then $g$ maps a continuous $L$-quasi-geodesic $\eta$ connecting $y$ to $x$ with $\eta(0) \in K\left(x, y, z ; m_{0}\right)$ to a continuous $L$ quasi-geodesic $g \eta$ connecting $b^{\prime}$ to $a^{\prime}$. Since $g(\eta(0)) \in K\left(a^{\prime}, b^{\prime}, g z ; m_{0}\right)$ we have

$$
\left|d\left(g \eta(0), x_{0}\right)-k / \chi\right| \leq m_{2}+\rho .
$$

Now let $x^{\prime} \in U(a), y^{\prime} \in U(b), z^{\prime} \in U(c)$ and let $g^{\prime} \in \Gamma$ be such that $g^{\prime} x^{\prime}=a^{\prime}$ $=g x, g^{\prime} y^{\prime}=b^{\prime}=g y$ and $\delta\left(g^{\prime} z^{\prime}, a^{\prime}\right) \in\left[e^{-k}, e^{-k+1}\right]$. Let $\eta^{\prime}$ be a continuous $L$-quasigeodesic connecting $y^{\prime}$ to $x^{\prime}$, with $\eta^{\prime}(0) \in B\left(x_{0}, m_{0}\right)$. As above, let $\gamma$ be a continuous $L$-quasi-geodesic connecting $b$ to $a$ with $\gamma(0) \in B\left(x_{0}, m_{0}\right)$ and let $\sigma<0$ be such that $d\left(x_{0}, \gamma(\sigma)\right)=R / \chi$. Then there are numbers $\tau, \tau^{\prime}<0$ such that $d(\eta(\tau), \gamma(\sigma)) \leq m_{1}$, $d\left(\eta^{\prime}\left(\tau^{\prime}\right), \gamma(\sigma)\right) \leq m_{1}$ and therefore $d\left(\eta(\tau), \eta^{\prime}\left(\tau^{\prime}\right)\right) \leq 2 m_{1}$. In particular, we have

$$
\left|d(\eta(0), \eta(\tau))-d\left(\eta^{\prime}(0), \eta^{\prime}\left(\tau^{\prime}\right)\right)\right| \leq 2 m_{0}+2 m_{1} .
$$

The images of $\eta, \eta^{\prime}$ under $g, g^{\prime}$ are continuous $L$-quasi-geodesics connecting $b^{\prime}$ to $a^{\prime}$. The estimate 97 is valid for $g^{\prime}$ as well and hence by hyperbolicity, the distances $d\left(g \eta(0), g^{\prime}\left(\eta^{\prime}(0)\right)\right), d\left(g(\eta(\tau)), g^{\prime}\left(\eta^{\prime}\left(\tau^{\prime}\right)\right)\right)$ are bounded from above by a universal constant $m_{3}>2 m_{2}$. Together we conclude that

$$
d\left(g^{-1} g^{\prime}(\eta(0)), \eta(0)\right) \leq 2 m_{3}, \quad d\left(g^{-1} g^{\prime}(\eta(\tau)), \eta(\tau)\right) \leq 2 m_{3} .
$$


Now if $R_{0}=R\left(x_{0}, 2 m_{3}\right)$ is as in the definition of a weakly acylindrical action, then for $R \geq \chi R_{0}$ and any $k \geq R$ the number of elements $g, g^{\prime} \in \Gamma$ with this property is bounded from above by a universal constant independent of $R$ and $k$. This shows that the action of $\Gamma$ on $\partial X$ satisfies the first property in the definition of a metrically proper action.

The second property in that definition follows from exactly the same argument. Namely, using the above notation, there is a number $\kappa>m_{0}(v)$ only depending on $v$ such that if $Z \subset \partial X$ is the set of all points whose distance to $U(a), U(b)$ is at least $v$ then there is a number $\tau_{0}>0$ such that for any $x \in U(a), y \in U(b)$ and $z \in Z$ the set $K(x, y, z)$ is contained in the ball of radius $\kappa>0$ about $x_{0}$. In other words, for any element $g \in \Gamma$ which maps a triple $(x, y, z) \in U(a) \times U(b) \times Z$ to a triple of points whose pairwise distances are bounded from below by $v$, the distance between $x_{0}$ and $g x_{0}$ is at most $\kappa$. The above considerations then show that we can find a number $\tilde{R}(v)>0$ depending on $v$ and some $\tilde{m}(v)>0$ such that the second requirement in the definition of a metrically proper action holds with these constants and for the action of $\Gamma$ on $\partial X$.

Recall from Section 3 the definition of a homeomorphism with north-south dynamics of a metric space of finite diameter. The statement of the next simple lemma is well known in the case that the hyperbolic space $X$ is proper; we include a short proof for the sake of completeness since we have not found a suitable reference for the general case.

Lemma 4.3. Let $X$ be a hyperbolic geodesic metric space and let $g$ be an isometry of $X$ such that for some $x \in X$ the map $k \mapsto g^{k} x$ is a quasi-isometric embedding of the integers into $X$. Then $g$ acts on $\partial X$ with north-south dynamics.

Proof. Let $g$ be an isometry of $X$ as in the lemma. Then the sequence $\left(g^{k} x\right)_{k \geq 0} \subset X$ converges to a point $a \in \partial X$, and the sequence $\left(g^{-k} x\right)_{k \geq 0} \subset X$ converges to a point $b \in \partial X-\{a\}$. The limit set of the infinite cyclic group $G$ generated by $g$ consists of the two points $a \neq b \in \partial X$, and these are fixed points for the action of $G$ on $\partial X$.

By hyperbolicity there is a number $m>0$ such that for every $\xi \in \partial X-\{a, b\}$ the closed set $K(a, b, \xi ; m) \subset X$ of all points in $X$ whose distance to each side of an $L$ quasi-geodesic triangle with vertices $a, b, \xi$ is at most $m$ is non-empty and its diameter $K(a, b, \xi ; m)$ is bounded independently of $\xi$. Since the assignment $k \mapsto g^{k}(x)$ is a quasiisometric embedding of the integers into $X$, we may assume by possibly enlarging $m$ that each of the sets $K(a, b, \xi ; m)$ intersects $Q=\left\{g^{k}(x) \mid k \in \mathbb{Z}\right\}$. Thus there is a number $l>0$ and for every $\xi \in \partial X-\{a, b\}$ there is some $\kappa(\xi) \in \mathbb{Z}$ such that the set $\left\{g^{\kappa}(x) \mid \kappa(\xi) \leq \kappa \leq \kappa(\xi)+l\right\}$ contains the intersection of $K(a, b, \xi ; m)$ with $Q$. Then $\left|\kappa\left(g^{j} \xi\right)-\kappa(\xi)-j\right| \leq l$ for all $j \in \mathbb{Z}$ and hence the set $D=\{\xi \in \partial X-\{a, b\} \mid 0 \leq$ $\kappa(\xi) \leq l\}$ does not contain $a, b$ in its closure and it satisfies $\bigcup_{j \in \mathbb{Z}} g^{j} D=\partial X-\{a, b\}$. Moroever, for any neighborhoods $U$ of $a$ and $V$ of $b$ there is a number $j>0$ such that $g^{j}(X-V) \subset U$ and $g^{-j}(X-U) \subset V$. Hence the isometry $g$ acts with north-south dynamics on $\partial X$.

Call an isometry of $X$ hyperbolic if it acts on $\partial X$ with north-south dynamics with respect to some fixed points $a \neq b$. The following corollary is immediate from Lemma 4.1, Lemma 4.2 and the remark after Proposition 3.2 in Section 3. We refer to [PR04] for a similar result for the group $S L(2, \mathbb{Z})$. 
Corollary 4.4. Let $\Gamma$ be a countable group which admits a weakly acylindrical isometric action on a hyperbolic geodesic metric space. Let $g_{1}, \ldots, g_{k} \in \Gamma$ be hyperbolic elements with ordered pairs of fixed points $\left(a_{i}, b_{i}\right)$. If the $\Gamma$-orbits of $\left(a_{i}, b_{i}\right),\left(b_{i}, a_{i}\right)$ are pairwise disjoint then for every $\left(q_{1}, \ldots, q_{k}\right) \in \mathbb{R}^{k}$ there is a quasi-morphism $\varphi$ for $\Gamma$ with $\lim _{\ell \rightarrow \infty} \varphi\left(g_{i}^{\ell}\right) / \ell=q_{i}$ for every $i \leq k$.

The limit set of an isometric action of a group $\Gamma$ on $X$ is the set of accumulation points in $\partial X$ of an orbit $\Gamma x(x \in X)$ of $\Gamma$; it does not depend on the orbit. A subgroup $\Gamma$ of Iso $(X)$ is called elementary if its limit set contains at most two points. The next result is Theorem A from the introduction.

Theorem 4.5. Let $\Gamma$ be a countable group which admits a non-elementary weakly acylindrical isometric action on a Gromov hyperbolic geodesic metric space $X$. Then the kernels of the natural homomorphisms $H_{b}^{2}(\Gamma, \mathbb{R}) \rightarrow H^{2}(\Gamma, \mathbb{R})$ and $H_{b}^{2}\left(\Gamma, \ell^{p}(\Gamma)\right) \rightarrow$ $H^{2}\left(\Gamma, \ell^{p}(\Gamma)\right)(1<p<\infty)$ are infinite-dimensional.

Proof. Let $\Gamma$ be a countable non-elementary weakly acylindrical subgroup of Iso $(X)$. By assumption, the limit set $\Lambda$ of $\Gamma$ contains at least three points. Then this limit set is a $\Gamma$ invariant closed subset of $\partial X$ without isolated points (see [GH90]). Our goal is to show that the action of $\Gamma$ on $\Lambda$ satisfies the assumptions in Theorem 3.3.

By Lemma 4.1 and Lemma 4.2, the action of $\Gamma$ on $\Lambda$ is weakly hyperbolic and the action of $\Gamma$ on the space of triples of pairwise distinct points in $\Lambda$ is metrically proper. Using Lemma 4.3 it is enough to show that $\Gamma$ contains a free subgroup $G$ with two generators which has the following additional properties.

(1) For some $x \in X$ the orbit map $g \in G \mapsto g x \in X$ is a quasi-isometric embedding of $G$ into $X$.

(2) There are infinitely many $g_{i} \in G(i \geq 0)$ such that the ordered pairs of fixed points of $g_{i}, g_{j}^{-1}$ are contained in pairwise distinct orbits of the action of $\Gamma$ on $\Lambda \times \Lambda$.

Note that the first property guarantees that there is a continuous $G$-equivariant embedding of the Gromov boundary $B$ of $G$ into $\Lambda$.

The existence of a free group $G$ with two generators and with property (1) above is immediate from the ping-pong lemma and the requirement that the group $\Gamma$ is nonelementary (cf. [GH90]).

Now let $e \neq g \in G$ and let $(a, b)$ be the ordered pair of fixed points of the action of $g$ on $\partial X$. Choose a closed subset of $\partial X$ which is contained in $X-\{a, b\}$ and is a fundamental domain $D$ for the action on $\partial X-\{a, b\}$ of the infinite cyclic subgroup of $G$ generated by $g$. Assume that there is a sequence $\left(a_{i}, b_{i}\right) \in \partial X \times \partial X$ contained in the $\Gamma$-orbit of $(a, b)$ with $\left(a_{i}, b_{i}\right) \rightarrow(a, b)$. Let $\delta$ be a Gromov distance on $\partial X$ and write $v=$ $\min \{\delta(a, b), \delta(\{a, b\}, D)\} / 4$. Let $R(v)>0$ be as in the definition of a metrically proper action for $\Gamma$ and let $U, V$ be the open $e^{-R(v)}$-neighborhoods of $a, b$ respectively. For sufficiently large $i$ we have $a_{i} \in U$ and $b_{i} \in V$. By our assumption, there are $h_{i} \in \Gamma$ such that $h_{i} a_{i}=a$ and $h_{i} b_{i}=b$. Then $h_{i}^{-1} g h_{i}$ is a hyperbolic isometry with fixed points $a_{i}, b_{i}$. Since a hyperbolic isometry fixes precisely two points in $\partial X$, the elements $h_{i}$ are pairwise distinct and the same is true for their compositions with an arbitrary power of $g$. Namely, 
otherwise there are $i \neq j$ and $l \in \mathbb{Z}$ such that $g^{l}=h_{i} h_{j}^{-1}$, which contradicts the fact that $(a, b)$ are fixed points for $g,\left(a_{i}, b_{i}\right) \neq\left(a_{j}, b_{j}\right)$ and $h_{i}$ is a homeomorphism. However, by the choice of $D$ there is for each $i>0$ some $k(i) \in \mathbb{Z}$ such that $g^{k(i)} h_{i} D \cap D \neq \emptyset$ and hence $g^{k(i)} h_{i}(U \times V \times D) \cap U \times V \times D \neq \emptyset$ for all sufficiently large $i$. This contradicts the assumption that the action of $\Gamma$ on the space of triples of pairwise distinct points in $\partial X$ is metrically proper.

As a consequence, for every ordered pair $(a, b)$ of fixed points of an element $e \neq g \in$ $G$ the $\Gamma$-orbit of $(a, b)$ is a discrete subset of $\partial X \times \partial X-\Delta$ (note that this fact has already been established in the proof of Proposition 3.2). Since on the other hand the sets of pairs of fixed points for the elements of $G$ are dense in $B \times B-\Delta$, there are infinitely many such pairs $\left(a_{i}, b_{i}\right)$ which are pairwise contained in distinct orbits under the action of $\Gamma$. Our argument also implies that we may in addition require that the ordered pairs $\left(a_{i}, b_{i}\right)$ are not contained in the $\Gamma$-orbit of $\left(b_{j}, a_{j}\right)$ for any $j$.

We use this fact to show that we can find infinitely many $g_{i} \in G$ with the property that the $\Gamma$-orbits of the ordered pairs of fixed points $\left(a_{i}, b_{i}\right),\left(b_{j}, a_{j}\right)$ of $g_{i}, g_{j}^{-1}$ are all disjoint (see the argument in [BF02]). Namely, choose two independent elements $g_{1}, g_{2} \in G$ which generate a free subgroup with the property that the ordered pairs of fixed points $\left(a_{1}, b_{1}\right),\left(b_{1}, a_{1}\right)$ of $g_{1}, g_{1}^{-1}$ are not contained in the $\Gamma$-orbit of the ordered pair of fixed points $\left(b_{2}, a_{2}\right)$ of $g_{2}^{-1}$. We may assume that the group generated by $g_{1}, g_{2}$ equals $G$ and that there is an $L$-quasi-isometric $G$-equivariant embedding $\rho$ of the Cayley graph $C G$ of $G$ into $X$ which induces an equivariant embedding of the Gromov boundary $B$ of $G$ into $\partial X$. Identify $C G$ with its image under this embedding. For $0 \ll n_{1} \ll m_{1} \ll n_{2} \ll m_{2}$ consider the element $f=g_{1}^{n_{1}} g_{2}^{m_{1}} g_{1}^{n_{2}} g_{2}^{m_{2}} \in G$. If $\gamma$ is the axis of $f$ in $C G$ and if $h \in \Gamma$ maps the ordered pair $(a, b)$ of fixed points for $f$ to $(b, a)$, then it maps the inverse $\rho(\gamma)^{-1}$ of $\rho(\gamma)$ into a uniformly bounded neighborhood of $\rho(\gamma)$. Now a fundamental domain for the action of $f$ on its axis $\gamma$ is composed of four arcs $\gamma_{1}, \ldots, \gamma_{4}$ where $\gamma_{1}$ is the geodesic arc in $C G$ connecting $e$ to $g_{1}^{n_{1}}, \gamma_{2}$ is the translate under $g_{1}^{n_{1}}$ of the geodesic arc connecting $e$ to $g_{2}^{m_{1}}$ etc. As a consequence, there is a subsegment of the axis of a conjugate of $g_{1}$ in $G$ whose length tends to infinity as $n_{1} \rightarrow \infty$ and which is mapped by $h$ into a uniformly bounded neigborhood of a subsegment of the axis of a conjugate of $g_{2}^{-1}$ (see [BF02]). For sufficiently large $n_{1}$ this violates the observation that the $\Gamma$-orbits of $\left(a_{i}, b_{1}\right),\left(b_{2}, a_{2}\right)$ are discrete and disjoint.

As a consequence, property (2) above holds for $G$ as well (cf. also the discussion in [BF02]). Thus the theorem is a consequence of Theorem 3.1.

\section{Applications}

In this section we derive some applications of Theorem A from the introduction. We begin with the proof of Corollary B from the introduction. For this let $S$ be an oriented surface of genus $g \geq 0$ with $m \geq 0$ punctures. We assume that $S$ is non-exceptional, i.e. that $3 g-3+m \geq 2$. The complex of curves $\mathcal{C}(S)$ for $S$ is the simplicial complex whose vertices are the free homotopy classes of essential simple closed curves on $S$, i.e. simple closed curves which are neither contractible nor freely homotopic into a puncture 
of $S$. The simplices in $\mathcal{C}(S)$ are spanned by collections of such curves which can be realized disjointly. Since $S$ is non-exceptional by assumption, the complex of curves is connected. If we equip each simplex in $\mathcal{C}(S)$ with the standard euclidean metric of sidelength one, then we obtain a length metric on $\mathcal{C}(S)$, and this length metric defines on $\mathcal{C}(S)$ the structure of a hyperbolic geodesic metric space. However, $\mathcal{C}(S)$ is not locally finite and hence this geodesic metric space is not locally compact (for all this see [MM99, B02, H07]). A description of its Gromov boundary is contained in [K199, H06].

The mapping class group $\mathcal{M}_{g, m}$ of $S$ is the group of isotopy classes of orientation preserving homeomorphisms of $S$. It acts as a group of isometries on the complex of curves $\mathcal{C}(S)$ of $S$. Bowditch [B03] showed that this action is weakly acylindrical. Thus we can apply Theorem 4.5 and deduce Corollary B from the introduction which extends the result of Bestvina and Fujiwara [BF02].

Proposition 5.1. Let $\Gamma$ be an arbitrary subgroup of $\mathcal{M}_{g, m}$. If $\Gamma$ is not virtually abelian then the group $H_{b}^{2}(\Gamma, \mathbb{R})$ is infinite-dimensional. If moreover $\Gamma$ does not contain a normal subgroup which virtually splits as a direct product of two infinite groups then for every $p \in(1, \infty)$ the group $H_{b}^{2}\left(\Gamma, \ell^{p}(\Gamma)\right)$ is infinite-dimensional as well.

Proof. Recall from [MP89] the classification of subgroups $\Gamma$ of $\mathcal{M}_{g, m}$. There are four cases:

(1) $\Gamma$ contains two independent pseudo-Anosov elements.

(2) The limit set of the action of $\Gamma$ on $\mathcal{C}(S)$ consists of precisely two points $a \neq b$.

(3) $\Gamma$ is finite.

(4) $\Gamma$ preserves a non-trivial system of pairwise disjoint essential simple closed mutually not freely homotopic curves on $S$.

The action of the mapping class group on $\mathcal{C}(S)$ is weakly acylindrical [B03] and hence the same is true for the action of an arbitrary subgroup $\Gamma$ of $\mathcal{M}_{g, m}$. If $\Gamma$ is as in case (1) above then the limit set of $\Gamma$ contains at least three points and therefore $\Gamma$ is a non-elementary subgroup of the isometry group of $\mathcal{C}(S)$. By Theorem 4.5 , the groups $H_{b}^{2}(\Gamma, \mathbb{R}), H_{b}^{2}\left(\Gamma, \ell^{p}(\Gamma)\right)$ are infinite-dimensional for every $p \in(1, \infty)$.

In case (2) above, each element of $\Gamma$ maps a quasi-geodesic connecting $a$ to $b$ into a uniformly bounded neighborhood of itself. Since the action of $\Gamma$ on $\mathcal{C}(S)$ is weakly acylindrical, the group $\Gamma$ is virtually cyclic (cf. the discussion in [BF02]).

In case (4) there is a maximal system $\mathcal{S}$ of pairwise disjoint essential simple closed non-mutually freely homotopic curves preserved by $\Gamma$. If we cut $S$ open along $\mathcal{S}$ and replace each boundary circle of the resulting bordered surface by a puncture then we obtain a possibly disconnected surface $S^{\prime}$ of finite type and of greater Euler characteristic. There is a natural homomorphism of $\Gamma$ onto a subgroup $\Gamma^{\prime}$ of the mapping class group of $S^{\prime}$. Its kernel $K$ is a free abelian group generated by multiple Dehn twists about the curves of this curve system. Thus by Theorem 12.4.2 of [M01] (see also Corollary 3.6 of [MS06]), the natural map $H_{b}^{2}\left(\Gamma^{\prime}, \mathbb{R}\right) \rightarrow H_{b}^{2}(\Gamma, \mathbb{R})$ is an isomorphism.

Let $S_{1}^{\prime}, \ldots, S_{p}^{\prime}$ be the connected components of $S^{\prime}$. An element $g \in \Gamma^{\prime}$ permutes the components of $S^{\prime}$. This means that there is a homomorphism $\kappa$ of $\Gamma^{\prime}$ into the group of permutations of $\{1, \ldots, p\}$ whose kernel is the normal subgroup $G$ of $\Gamma$ of all elements 
which fix each component $S_{i}^{\prime}$. Thus there is an exact sequence

$$
0 \rightarrow G \rightarrow \Gamma^{\prime} \rightarrow Q \rightarrow 0
$$

where $Q$ is a finite group. This sequence induces an exact sequence [M01]

$$
\cdots \rightarrow H_{b}^{2}(Q, \mathbb{R}) \rightarrow H_{b}^{2}\left(\Gamma^{\prime}, \mathbb{R}\right) \rightarrow H_{b}^{2}(G, \mathbb{R}) \rightarrow H_{b}^{3}(Q, \mathbb{R}) \rightarrow \cdots
$$

Since the group $Q$ is finite, its bounded cohomology with real coefficients is finite dimensional and therefore we conclude that $H_{b}^{2}\left(\Gamma^{\prime}, \mathbb{R}\right)$ is infinite-dimensional if and only if this is the case for $H_{b}^{2}(G, \mathbb{R})$.

For $i \leq p$ denote by $G_{i}$ the projection of $G$ to a subgroup of the mapping class group of $S_{i}^{\prime}$. If $G_{i}$ preserves a non-trivial system $\mathcal{S}_{i}$ of pairwise disjoint essential simple closed non-mutually freely homotopic curves on $S_{i}^{\prime}$ then the $\Gamma^{\prime}$-translates of this system is a $\Gamma^{\prime}$-invariant curve system on $S^{\prime}$ which lifts to a $\Gamma$-invariant curve system on $S$ strictly containing $\mathcal{S}$. This contradicts the maximality of the system $\mathcal{S}$.

An exceptional component $S_{i}^{\prime}$ of $S^{\prime}$ is either a thrice punctured sphere with finite mapping class group, or a once punctured torus or a four times punctured sphere with word hyperbolic mapping class group. Therefore either $\Gamma^{\prime}$ and hence $\Gamma$ is virtually abelian, or after reordering, the group $G_{1}$ admits a weakly acylindrical action as a non-elementary group of isometries on a hyperbolic geodesic metric space. In particular, if $\Gamma$ is not virtually abelian then the second bounded cohomology group $H_{b}^{2}\left(G_{1}, \mathbb{R}\right)$ is infinite-dimensional.

Let $R$ be the kernel of the homomorphism $G \rightarrow G_{1}$. Then we have an exact sequence

$$
0 \rightarrow R \rightarrow G \rightarrow G_{1} \rightarrow 0
$$

Since necessarily $H_{b}^{1}(R, \mathbb{R})=0$ (see [M01]) we deduce from the induced exact sequence of bounded cohomology groups that $H_{b}^{2}(G, \mathbb{R})$ is infinite-dimensional if this is the case for $H_{b}^{2}\left(G_{1}, \mathbb{R}\right)$. In other words, either $\Gamma$ is virtually abelian or the second bounded cohomology group $H_{b}^{2}(\Gamma, \mathbb{R})$ is infinite-dimensional.

We are left with investigating the groups $H_{b}^{2}\left(\Gamma, \ell^{p}(\Gamma)\right)$. Assume that $\Gamma$ is not virtually abelian. Then the group $G$ is infinite. Thus using the above notations, if the kernel $K$ of the natural projection $\pi: \Gamma \rightarrow \Gamma^{\prime}$ is non-trivial, then the normal subgroup $\pi^{-1}(G)$ of $\Gamma$ splits as a direct product of two infinite groups. Hence as before, we may assume that $\Gamma=\Gamma^{\prime}$. Then $H_{b}^{2}\left(\Gamma, \ell^{p}(\Gamma)\right)$ is infinite-dimensional if this is the case for $H_{b}^{2}\left(G, \ell^{p}(G)\right)$. Namely, if the centralizer $Z_{\Gamma}(G)$ of $G$ in $\Gamma$ is infinite then the center of $G$ is infinite and hence either $G$ is virtually abelian or $G$ splits as a direct product of two infinite groups. Thus we may assume that $Z_{\Gamma}(G)$ is finite. Then every function $f \in \ell^{p}(G)$ which is invariant under the action of the finite center of $G$ defines a function in the $G$-module $\ell^{p}(\Gamma)^{Z_{\Gamma}(G)}$ of $Z_{\Gamma}(G)$-invariant points in $\ell^{p}(\Gamma)$ which vanishes outside of $G Z_{\Gamma}(G)$. It follows that the second bounded cohomology group $H_{b}^{2}\left(G, \ell^{p}(\Gamma)^{Z_{\Gamma}(G)}\right)$ is infinite-dimensional.

The finite group $Q$ as in the exact sequence 10 admits an isometric action on $H_{b}^{2}\left(G, \ell^{p}(\Gamma)^{Z_{\Gamma}(G)}\right)$ induced from the action of $Q$ on $G$ by conjugation (Corollary 8.7.3 of [M01]). Unsing the explicit form of this action we conclude that the subspace of $H_{b}^{2}\left(G, \ell^{p}(\Gamma)^{Z_{\Gamma}(G)}\right)$ of elements which are fixed by $Q$ is infinite-dimensional if this is the 
case for $H_{b}^{2}\left(G, \ell^{p}(G)\right)$. On the other hand, since the group $G$ is infinite by assumption, there is no non-zero $G$-invariant vector in $\ell^{p}(\Gamma)$ and hence by the Hochschild-Serre spectral sequence for bounded cohomology (Theorem 12.0.3 of [M01]), the second bounded cohomology $H_{b}^{2}\left(\Gamma, \ell^{p}(\Gamma)\right)$ is infinite-dimensional if this is the case for $H_{b}^{2}\left(G, \ell^{p}(G)\right)$.

Let $N_{j}$ be the kernel of the projection of $G$ into a subgroup of the mapping class group of $S^{\prime}-S_{j}^{\prime}$. Then $N_{j}$ consists of mapping classes which act trivially on $S_{i}$ for all $i \neq j$. For $i \neq j$, the groups $N_{i}, N_{j}$ only intersect in the identity and commute. Hence if they are infinite for some $i \neq j$, then $G$ contains a normal subgroup which is the direct product of two infinite groups. The smallest normal subgroup of $\Gamma$ containing $N_{i}, N_{j}$ contains the direct product of $N_{i}, N_{j}$ as a subgroup of finite index, i.e. this normal subgroup virtually splits as a direct product. Thus for the purpose of the proposition we may assume after reordering that $N_{i}$ is finite for all $i>1$.

Consider first the case that $N_{1}$ is infinite. Denote as before by $R$ the kernel of the natural projection $G \rightarrow G_{1}$ into the mapping class group of $S_{1}^{\prime}$. The subgroup of $G$ generated by $N_{1}, R$ is normal and the direct product of $N_{1}$ and $R$. Therefore as above, if $\Gamma$ does not contain a normal subgroup which virtually splits as a direct product of two infinite groups then $R$ is finite, and the quotient group $G / R$ can naturally be identified with the group $G_{1}$.

Assume that this holds true. By Theorem 4.5 and the assumption that $G$ is not virtually abelian, the second bounded cohomology group $H_{b}^{2}\left(G_{1}, \ell^{p}\left(G_{1}\right)\right)$ is infinitedimensional for every $p \in(1, \infty)$. Now the group $R$ is finite and hence averaging over the orbits of the action of $R$ shows that $\ell^{p}\left(G_{1}\right)$ as a $G_{1}$-module can naturally be identified with the $G_{1}$-module $\ell^{p}(G)^{R}$ of all $R$-invariant points in $\ell^{p}(G)$. As a consequence, the group $H_{b}^{2}\left(G_{1}, \ell^{p}(G)^{R}\right)$ is infinite-dimensional, and therefore from the HochschildSerre spectral sequence (Theorem 12.0.3 of [M01]) we deduce that the same is true for $H_{b}^{2}\left(G, \ell^{p}(G)\right)$. We deduce that if $N_{1}$ is infinite and if $\Gamma$ does not contain a normal subgroup which virtually splits as a direct product then $H_{b}^{2}\left(G, \ell^{p}(G)\right)$ is infinite-dimensional as claimed.

Finally, we have to consider the case that $N_{1}$ is finite, i.e. that the kernel of the natural projection of $G$ to a subgroup of the mapping class group of $S_{2}^{\prime} \cup \cdots \cup S_{p}^{\prime}$ is finite. By the above considerations, for every $p \in(1, \infty)$ the group $H_{b}^{2}\left(G, \ell^{p}(G)\right)$ is infinitedimensional if this is the case for $H_{b}^{2}\left(G / N_{1}, \ell^{p}\left(G / N_{1}\right)\right)$. Since $\Gamma$ contains a normal subgroup which virtually splits as a direct product if this is the case for $G / N_{1}$, an application of the above considerations to the group $G / N_{1}$ yields inductively the following. Either $\Gamma$ contains a normal subgroup which virtually splits as a direct product or $H_{b}^{2}\left(\Gamma, \ell^{p}(\Gamma)\right)$ is infinite-dimensional. This shows the proposition.

Following [MS06], we denote by $\mathcal{C}_{\text {geom }}$ the class of countable groups which admit a non-elementary weakly acylindrical isometric action on some hyperbolic metric space. Examples of such groups include.

- Word hyperbolic groups which are not virtually abelian.

- Any subgroup of the mapping class group of an oriented surface of finite type and negative Euler characteristic not preserving any essential multicurve, e.g. the Torelli group. 
- Any countable group which admits a non-elementary isometric action on a (not necessarily locally finite) tree which is proper on the edges.

This class also contains a large family of relatively hyperbolic groups. In fact, it seems that all geometrically finite relatively hyperbolic groups in the sense of Bowditch (see [Y04] for a detailed discussion of those groups) are contained in $\mathcal{C}_{\text {geom }}$.

For a locally compact $\sigma$-compact topological group $G$ define a lattice in $G$ to be a discrete subgroup $\Gamma$ of $G$ such that $G / \Gamma$ admits a finite $G$-invariant measure. If $G=$ $G_{1} \times G_{2}$ is any non-trivial direct product with locally compact $\sigma$-compact and noncompact factors then we call a lattice $\Gamma$ in $G$ irreducible if the projection of $\Gamma$ into each of the factors is dense. The following lemma is part (vi) of Proposition 7.13 in [MS06] and follows from the work of Burger and Monod [BM02].

Lemma 5.2. Let $\Gamma$ be an irreducible lattice in a product $G=G_{1} \times G_{2}$ of locally compact $\sigma$-compact non-compact groups. Then $H_{b}^{2}\left(\Gamma, \ell^{2}(\Gamma)\right)=0$.

We use Lemma 5.2 and the results of Monod and Shalom [MS06] to show

Corollary 5.3. A group $\Gamma \in \mathcal{C}_{\text {geom }}$ is not measure equivalent to any finitely generated irreducible lattice in either a simple Lie group of higher rank or in a product of two locally compact $\sigma$-compact and non-compact topological groups.

Proof. By Theorem 4.5, for every $\Gamma \in \mathcal{C}_{\text {geom }}$ the group $H_{b}^{2}\left(\Gamma, \ell^{2}(\Gamma)\right)$ is non-trivial. Then Corollary 7.8 of [MS06] shows that $H_{b}^{2}\left(\Lambda, \ell^{2}(\Lambda)\right) \neq\{0\}$ for every countable group $\Lambda$ which is measure equivalent to $\Gamma$.

Now by Lemma 5.2, if $\Lambda$ is an irreducible lattice in a product $G_{1} \times G_{2}$ of locally compact $\sigma$-compact non-compact groups then $H_{b}^{2}\left(\Lambda, \ell^{2}(\Lambda)\right)=\{0\}$. If $\Lambda$ is a lattice in a simple Lie group of non-compact type and higher rank then the vanishing of the second bounded cohomology group $H_{b}^{2}\left(\Lambda, \ell^{2}(\Lambda)\right.$ ) is due to Monod and Shalom (Theorem 1.4 in [MS04]). Thus in both cases, the group $\Lambda$ is not measure equivalent to $\Gamma$. (Note however that for lattices $\Lambda$ in simple Lie groups of higher rank a much stronger result is due to Furman [Fu99a, Fu99b]: Every countable group which is measure equivalent to $\Lambda$ is commensurable to $\Lambda$.)

Corollary $\mathrm{C}$ from the introduction is now immediate from Corollary 5.3 and Proposition 5.1 .

We finish the paper by mentioning two results of Monod and Shalom [MS06] which are closely related to this work.

Theorem 5.4. A countable group containing an infinite amenable normal subgroup is not measure equivalent to a group in $\mathcal{C}_{\text {geom }}$.

Another consequence is Monod and Shalom's striking rigidity result for actions of products (Theorem 1.8 of [MS06]).

Theorem 5.5. Let $\Gamma_{1}, \Gamma_{2}$ be torsion free groups in $\mathcal{C}_{\text {geom }}, \Gamma=\Gamma_{1} \times \Gamma_{2}$ and let $(X, \mu)$ be an irreducible probability $\Gamma$-space. Let $\Lambda$ be any torsion free countable group and let 
$(Y, v)$ be any mildly mixing probability $\Lambda$-space. If the $\Gamma$-action and the $\Lambda$-action are orbit equivalent, then both groups as well as the actions are commensurable.

There is also a version of Theorem A for closed groups of isometries of proper hyperbolic spaces and their continuous bounded cohomology [H05].

Acknowledgments. I am very grateful to Yehuda Shalom for pointing out an error in an earlier version of this paper and for additional valuable suggestions. I also thank the anonymous referee for useful comments.

This research was partially supported by Sonderforschungsbereich 611 .

\section{References}

[A94] Adams, S.: Boundary amenability for word hyperbolic groups and an application to smooth dynamics of simple groups. Topology 33, 765-783 (1994) Zbl 0838.20042 MR 1293309

[BG88] Barge, J., Ghys, E.: Surfaces et cohomologie bornée. Invent. Math. 92, 509-526 (1988) Zbl 0641.55015 MR 0939473

[BF02] Bestvina, M., Fujiwara, K.: Bounded cohomology of subgroups of mapping class groups. Geom. Topol. 6, 69-89 (2002) Zbl 1021.57001 MR 1914565

[B02] Bowditch, B.: Intersection numbers and the hyperbolicity of the curve complex. J. Reine Angew. Math. 598, 105-129 (2006) Zbl pre05080523

[B03] Bowditch, B.: Tight geodesics in the curve complex. Ivent Math. 171, 281-300 (2008) Zbl pre05248088 MR 2367021

[BH99] Bridson, M., Haefliger, A.: Metric Spaces of Non-Positive Curvature. Springer, Berlin (1999) Zbl 0988.53001 MR 1744486

[Br81] Brooks, R.: Some remarks on bounded cohomology. In: Riemann Surfaces and Related Topics (Stony Brook, 1978), Ann. of Math. Stud. 97, Princeton Univ. Press, 55-63 (1981) Zbl 0457.55002 MR 0624804

[BM99] Burger, M., Monod, N.: Bounded cohomology of lattices in higher rank Lie groups. J. Eur. Math. Soc. 1, 199-235 (1999) Zbl 0932.22008 MR 1694584

[BM02] Burger, M., Monod, N.: Continuous bounded cohomology and applications to rigidity theory. Geom. Funct. Anal. 12, 219-280 (2002) Zbl 1006.22010 MR 1911660

[FM98] Farb, B., Masur, H.: Superrigidity and mapping class groups. Topology 37, 1169-1176 (1998) Zbl 0946.57018 MR 1632912

[F98] Fujiwara, K.: The second bounded cohomology of a group acting on a Gromov hyperbolic space. Proc. London Math. Soc. 76, 70-94 (1998) Zbl 0891.20027| MR 1476898

[Fu99a] Furman, A.: Gromov's measure equivalence and rigidity of higher rank lattices. Ann. of Math. 150, 1059-1081 (1999) Zbl 0943.22013 MR 1740986

[Fu99b] Furman, A.: Orbit equivalence rigidity. Ann. of Math. 150, 1083-1108 (1999) Zbl 0943.22012 MR 1740985

[GH90] Ghys, E., de la Harpe, P.: Sur les groupes hyperboliques d'après Mikhael Gromov. Birkhäuser, Boston (1990) Zbl 0731.20025 MR 1086648

[G83] Gromov, M.: Volume and bounded cohomology. Inst. Hautes Études Sci. Publ. Math. 56, 5-99 (1983) Zbl 0516.53046 MR 0686042

[G93] Gromov, M.: Asymptotic Invariants of Infinite Groups. London Math. Soc. Lecture Note Ser. 182, Cambridge Univ. Press (1993) Zbl 0841.20039 MR 1253544 
[H97] Hamenstädt, U.: Cocycles, Hausdorff measures and cross ratios. Ergodic Theory Dynam. Systems 17, 1061-1081 (1997) Zbl 0906.58035 MR 1477033

[H99] Hamenstädt, U.: Cocycles, symplectic structures and intersection. Geom. Funct. Anal. 9, 90-140 (1999) Zbl 0951.37007 MR 1675892

[H05] Hamenstädt, U.: Isometry groups of proper hyperbolic spaces. arXiv:math.GR/0507608

[H06] Hamenstädt, U.: Train tracks and the Gromov boundary of the complex of curves. In: Spaces of Kleinian Groups and Hyperbolic 3-Manifolds, Y. Minsky et al. (eds.), London Math. Soc. Lecture Note 329, Cambridge Univ. Press, 187-207 (2006) Zbl pre05056501 MR 2258749

[H07] Hamenstädt, U.: Geometry of the complex of curves and of Teichmüller space. In: Handbook of Teichmüller Theory, Vol. 1, A. Papadopoulos (ed.), Eur. Math. Soc., 447-467 (2007) MR 2349677

[HK95] Hasselblatt, B., Katok, A.: Introduction to the Modern Theory of Dynamical Systems. Cambridge Univ. Press, Cambridge (1995) Zbl 0878.58020 MR 1326374

[I87] Ivanov, N. V.: Foundations of the theory of bounded cohomology. J. Soviet Math. 37, 1090-1115 (1987) Zbl 0612.55006 MR 0806562

[Ka03] Kaimanovich, V.: Double ergodicity of the Poisson boundary and applications to bounded cohomology. Geom. Funct. Anal. 13, 852-861 (2003) Zbl 1027.60038 MR 2006560

[KM96] Kaimanovich, V., Masur, H.: The Poisson boundary of the mapping class group. Invent. Math. 125, 221-264 (1996) Zbl 0864.57014 MR 1395719

[K06] Kida, Y.: Measure equivalence rigidity of the mapping class group. Ann. of Math., to appear

[K199] Klarreich, E.: The boundary at infinity of the curve complex and the relative Teichmüller space. Preprint (1999)

[MM99] Masur, H., Minsky, Y.: Geometry of the complex of curves I: Hyperbolicity. Invent. Math. 138, 103-149 (1999) Zbl 0941.32012 MR 1714338

[MP89] McCarthy, J., Papadopoulos, A.: Dynamics on Thurston's sphere of projective measured foliations. Comment. Math. Helv. 64, 133-166 (1989) Zbl 0681.57002 MR 0982564

[MMS04] Mineyev, I., Monod, N., Shalom, Y.: Ideal bicombings for hyperbolic groups and applications. Topology 43, 1319-1344 (2004) Zbl pre02117933 MR 2081428

[M01] Monod, N.: Continuous Bounded Cohomology of Locally Compact Groups. Lecture Notes in Math. 1758, Springer (2001) Zbl 0967.22006 MR 1840942

[MS04] Monod, N., Shalom, Y.: Cocycle superrigidity and bounded cohomology for negatively curved spaces. J. Diff. Geom. 67, 395-456 (2004) Zbl pre05033775 MR 2153026

[MS06] Monod, N., Shalom, Y.: Orbit equivalence rigidity and bounded cohomology. Ann. of Math. 164, 825-878 (2004)

[PR04] Polterovich, L., Rudnik, Z.: Stable mixing for cat maps and quasi-morphisms of the modular group. Ergodic Theory Dynam. Systems 24, 609-619 (2004) Zbl 1071.37019 MR 2054053

[Y04] Yaman, A.: A topological characterization of relatively hyperbolic groups. J. Reine Angew. Math. 566, 41-89 (2004) Zbl 1043.20020 MR 2039323

[Z84] Zimmer, R.: Ergodic Theory and Semisimple Groups. Birkhäuser, Boston (1984) Zbl 0571.58015 MR 0776417

[Z91] Zimmer, R.: Groups generating transversals to semisimple Lie group actions. Israel J. Math. 73, 151-159 (1991) Zbl 0756.28013 MR 1135209 